\title{
Vlasov analysis of microbunching instability for magnetized beams
}

\author{
C.-Y. Tsai, ${ }^{1, *}$ Ya. S. Derbenev, ${ }^{2}$ D. Douglas, ${ }^{2}$ R. $\mathrm{Li}^{2},{ }^{2}$ and C. Tennant ${ }^{2}$ \\ ${ }^{1}$ Department of Physics, Virginia Tech, Blacksburg, Virginia 24061, USA \\ ${ }^{2}$ Jefferson Laboratory, Newport News, Virginia 23606, USA
}

(Received 17 January 2017; published 19 May 2017)

\begin{abstract}
For a high-brightness electron beam with high bunch charge traversing a recirculation beam line, coherent synchrotron radiation and space charge effects may result in microbunching instability (MBI). Both tracking simulation and Vlasov analysis for an early design of a circulator cooler ring (CCR) for the Jefferson Lab Electron Ion Collider (JLEIC) reveal significant MBI [Ya. Derbenev and Y. Zhang, Proceedings of the Workshop on Beam Cooling and Related Topics, COOL'09, Lanzhou, China, 2009 (2009), FRM2MCCO01]. It is envisioned that the MBI could be substantially suppressed by using a magnetized beam. In this paper we have generalized the existing Vlasov analysis, originally developed for a nonmagnetized beam (or transversely uncoupled beam), to the description of transport of a magnetized beam including relevant collective effects. The new formulation is then employed to confirm prediction of microbunching suppression for a magnetized beam transport in the recirculation arc of a recent JLEIC energy recovery linac (ERL) based cooler design for electron cooling. It is found that the smearing effect in the longitudinal beam phase space originates from the large transverse beam size as a nature of the magnetized beams and becomes effective through the $x-z$ correlation when the correlated distance is larger than the microbunched scale. As a comparison, MBI analysis of the early design of JLEIC CCR is also presented in this paper.
\end{abstract}

DOI: 10.1103/PhysRevAccelBeams.20.054401

\section{INTRODUCTION AND MOTIVATION}

Beam quality preservation is of general concern in delivering a high-brightness electron beam through a transport line or recirculation arc in the design of modern accelerators. During high-brightness beam transport, initial small density modulations can be converted into energy modulations due to short-ranged wakefields or highfrequency impedances. Then, the energy modulations can be transformed back to density counterparts downstream in dispersive regions. The density-energy conversion, if forming a positive feedback, can result in the enhancement of modulation amplitudes. This has been known as the microbunching instability (MBI) (see, for example, Refs. [1-3]). MBI has been one of the most challenging issues associated with beam line designs such as magnetic bunch compressor chicanes for free-electron lasers or linear colliders. Moreover, it also poses difficulties in the design of transport lines for recirculating or energyrecovery-linac (ERL) machines. Any dominant source of beam performance limitations in such a high-brightness electron beam transport system must be carefully examined in order to preserve beam phase-space quality. Among

jcytsai@vt.edu

Published by the American Physical Society under the terms of the Creative Commons Attribution 4.0 International license. Further distribution of this work must maintain attribution to the author(s) and the published article's title, journal citation, and DOI. those, we have already known the longitudinal space charge force (LSC) and coherent synchrotron radiation (CSR) can, in particular, drive MBI. The LSC effect stems from upstream ripples on top of the longitudinal charge density, and can generate an amount of energy modulation when the beam traverses along a section of a beam line. When the beam encounters bending, CSR due to electron coherent radiation emission inside a bend can have a significant effect upon further amplifying the induced density modulations downstream the recirculation arc. Even without CSR, a recirculation arc with multiple (usually several to tens of) bending dipoles can convert the upstream accumulated energy modulation into density modulation, resulting in deleterious phase space degradation. A typical transport line in a recirculated machine can have a long linac or straight section and a large number of bending dipoles, and thus can potentially incubate such density-energy conversion along the beam line. The successive accumulation and conversion mechanism between density and energy modulations can result in significant microbunching amplification.

The motivation of this work originates from the early design of the circulator cooler ring (CCR) [4] for the Jefferson Lab Electron Ion Collider (JLEIC) [5]. Utilizing the conventional electron cooling scheme, the goal of the JLEIC cooler design is to achieve a significant reduction of the six-dimensional ion beam emittance and to deliver the beam with small spot size at the interaction point for high luminosities. The cooler design thus serves as a critical 
technology element in delivering high luminosities over a broad center-of-mass energy range in JLEIC. In addition to the same longitudinal velocity (or Lorentz factor) of the electron beam as that of an ion beam and satisfying the optics matching condition in the cooling solenoid section, general requirements of electron beams for efficient electron cooling are (1) high bunch charge, (2) low beam temperature (or small electron beam emittance and energy spread), and (3) large enough transverse beam size in order to cool the traversing ion beams. The cooling electron beam then features a high peak bunch current and low energy (usually $\sim 55 \mathrm{MeV}$ to cool the proton beam at $100 \mathrm{GeV}$ ). These usually set stringent requirements on electron beam brightness because the high peak bunch current and low energy would enhance the collective interaction, and small beam emittance or energy spread would weaken Landau damping or the phase-space smearing effect.

Both tracking simulations [6,7] and Vlasov analysis [8-10] have shown that MBI is a serious concern for the early CCR design. The one-turn steady-state CSR microbunching gain (to be defined later) is found to be up to 4000 at the modulation wavelength of $360 \mu \mathrm{m}$ and is even higher when CSR transient or LSC effects are included. To be specific, this is mainly due to the high bunch charge $(\sim 2 \mathrm{nC})$ and relatively low energy $(\sim 55 \mathrm{MeV})$ of the cooling beam circulating in the CCR and because of ineffective Landau damping due to small beam emittance ( $\sim 3 \mu \mathrm{m}$, normalized) and small relative energy spread $\left(\sim 10^{-4}\right)$. Mitigation of MBI thus becomes an issue for a high-brightness beam transport in recirculating machines.

Several mitigation schemes have been proposed in the literature for different machine configurations and can be in general divided into two categories: those addressing the transport lattice optics, and those directed at the transported beam. For the former aspect, the optics impact of beam line lattice designs on MBI has been recently investigated (see, for example, Refs. [11-16]). In those beam line designs, the beam is assumed transversely uncoupled, i.e. nonmagnetized. For the latter aspect, Derbenev (see, for example, Refs. $[17,18])$ had proposed using a magnetized beam to improve electron cooling performance and to mitigate collective effects [19]. A magnetized beam in general features nonzero canonical angular momentum, thus considered to be a transversely coupled beam. Concerning why the JLEIC cooler design considers using a magnetized beam for cooling, the track of thought can be clarified as follows: the last two items of the aforementioned requirements (small beam emittance and large enough beam size) for efficient electron cooling seem to be in contradiction, at least for nonmagnetized beams. For a magnetized beam, it can be however admitted: the key to efficient electron cooling lies in the requirement of different descriptions of beam emittance for a magnetized beam from that for a nonmagnetized beam.
Through a coordinate transformation from a Cartesian coordinate frame to a beam rotating frame, the transverse intrinsic beam spread can be characterized by Larmor and drift emittances, $\varepsilon_{L}$ and $\varepsilon_{d}$, respectively [20]. The transverse $4 \mathrm{D}$ emittance is evaluated as the geometric mean $\varepsilon_{4 D}=\sqrt{\varepsilon_{L} \varepsilon_{d}}$. In Refs. [21-23], the electron cooling efficiency can be greatly improved by employing a magnetized beam. The reason is that, in a strong magnetic field, the transverse degree of freedom of a magnetized electron beam motion does not take part in the energy exchange, because collisions are adiabatically slow relative to the cyclotron oscillations. That is to say, the electron beam temperature is indeed determined by the (smaller) Larmor emittance $\varepsilon_{L}$, instead of the total emittance $\varepsilon_{4 D}$ or the conventional (Cartesian) emittance $\varepsilon_{x, y}$. The transverse beam size during transport is then related to the drift emittance $\varepsilon_{d}$. In this regard, the low temperature and large transverse size of the beam may not conflict for a magnetized beam. The (larger) drift emittance results in larger transverse beam size and, together with small Larmor emittance or low electron temperature, can effectively enhance the cooling efficiency. This clarifies the advantage of using a magnetized beam for electron cooling considered in the JLEIC.

A magnetized beam cooling was found to be an extremely useful technique in obtaining high-brightness hadron beams with low longitudinal momentum spread [22]. Another advantage of using magnetized beams has also been suggested, because it was believed to mitigate some collective effects such as space charge [24] and MBI (our primary focus in this paper) because of its relatively larger transverse beam size. A magnetized beam can be generated by immersing the cathode in an axial solenoid magnetic field and thus features a nonzero angular momentum. In general, the magnetized beam is a transversely coupled beam.

Now that the magnetized beam has a promising yet qualitative feature of mitigating collective effects, for our purpose we want to confirm its effectiveness on MBI using a more quantitative model. There are many challenges in the theoretical study of MBI for a magnetized beam. In theory, to the best of our knowledge, there is not yet a linear Vlasov formalism addressing the microbunching instability for the transversely coupled beam transport through a beam line including relevant collective effects. In numerical simulation, particle tracking simulation (see, for example, [2]) with inclusion of relevant collective effects can be valuable for beam dynamics studies. It allows realistic particle beam distribution and a general beam line lattice, but also requires careful treatment of various numerical parameters to ensure numerical convergence before the reliable results are obtained, in particular for the microbunched phase space dynamics. The timedomain treatment turns out to be considerably challenging when MBI becomes severe, e.g. for the early design of the 
JLEIC CCR (see Sec. V). Usually a large number of simulation particles and long computation time are required for reaching convergent results of microbunching gains, and strenuous efforts are needed to do parametric studies for machine designs or optimization in order to minimize MBI. In addition to microbunching dynamics, the numerical setup needs to take care of transverse angular momentum of the beam from its origination to preservation during beam transport. In practice, many other issues remain to be solved regarding how a magnetized beam can be generated with both high bunch charge and low temperature and how it can be transported while preserving outstanding beam phase space quality with its magnetization until the cooling section and so on. In this paper we are interested in the aforementioned theory and simulation parts, where we have generalized the existing linear Vlasov analysis, originally developed for a nonmagnetized beam (or transversely uncoupled beam) [3,4], to the description of transport of a magnetized beam including relevant collective effects. We also developed a new semianalytical Vlasov solver for this particular feature of the beam.

In the remainder of this paper, we shall introduce the single-particle beam optics transport and the concept of beam sigma matrix in Sec. II. To characterize the general feature of a magnetized (or transversely coupled) beam, we use the beam sigma matrix, although a set of generalized Twiss (or Courant-Snyder) parameters can do the same thing in another way; see e.g. Ref. [25]. Those who have been familiar with the transition from single-particle optics to multi-particle optics using beam sigma matrix and its diagonalization can skim this section. In Sec. III we derive the integral equations, which govern microbunching for a general transverse coupled beam in a single-pass system. In many situations, the coasting beam model serves as an excellent approximation to further simplify the subsequent semianalytical calculations. We quantify the microbunching phenomena by calculating both the density and energy modulations of a beam along a transport line. Here our formulation of MBI for a magnetized beam is an extension of the formulation for an uncoupled beam. For a transversely uncoupled beam and linear optics transport, the governing equation for density modulation is reduced to that derived by Heifets, Stupakov, and Krinsky [3] and Huang and Kim [4]. Section IV briefly summarizes the impedance models used to simulate CSR and LSC induced MBI. In Sec. V, we apply the results obtained in the previous sections to a specialized recirculation arc for magnetized beam transport in our recent ERL cooler design for JLEIC. In the same section, as a comparison, MBI analysis of the early design of the JLEIC CCR for a nonmagnetized beam is also presented. In spite of the different beam line designs and distinct natures of the circulating beams for the CCR and ERL cooler ring, it still serves informative comparison in view of microbunching dynamics. The underlying physics of effective suppression of MBI in a magnetized beam transport is discussed. We also benchmark our semianalytical Vlasov solutions against particle tracking by ELEGANT [26]. Both approaches are in good agreement. In the last section, Sec. VI, the results of the paper are summarized.

\section{BEAM OPTICS OF A TRANSPORT LINE}

We begin by defining the six-dimensional phase space coordinate as

$$
\mathbf{X}(s)=\left(x, x^{\prime}, y, y^{\prime}, z, \delta ; s\right)^{T}
$$

where $x$ and $y$ are transverse (horizontal/radial) and vertical positions, $x^{\prime}$ and $y^{\prime}$ are the corresponding angular divergences, $\left({ }^{\prime}\right) \equiv d / d s$, and $z$ and $\delta \equiv \Delta E / E$ are the (local) longitudinal coordinate and energy deviation (assuming $z>0$ for the bunch head). All these quantities are measured with respect to the reference particle and are a function of the (global) longitudinal path coordinate, $s$. The superscript $T$ indicates the transpose operator. For simplicity, we assume the beam energy is constant throughout the analysis. For the convenience of subsequent discussion, we also define two subsets of $\mathbf{X}$ as

$$
\begin{aligned}
& \mathbf{X}_{2 D}(s)=\left(x, x^{\prime} ; s\right)^{T}, \\
& \mathbf{X}_{4 D}(s)=\left(x, x^{\prime}, y, y^{\prime} ; s\right)^{T} .
\end{aligned}
$$

To study the single-particle beam optics, we introduce the (unperturbed) Hamiltonian as [27]

$$
\begin{aligned}
\bar{H}= & -(1+\delta)\left(1+\frac{x}{\rho_{x}}+\frac{y}{\rho_{y}}\right)\left(1-\frac{x^{\prime 2}}{2}-\frac{y^{\prime 2}}{2}\right) \\
& -\frac{e A_{s}}{p_{0}}\left(1+\frac{x}{\rho_{x}}+\frac{y}{\rho_{y}}\right)
\end{aligned}
$$

where $\rho_{x}$ and $\rho_{y}$ are bending radii of horizontal and vertical dipoles, respectively. $p_{0}=e B_{y, x} \rho_{x, y}$ is the total momentum of the reference particle with $e$ the unit charge and $B_{y, x}$ are respectively the transverse vertical and horizontal dipole fields. $A_{s}$ is the longitudinal component of the vector potential. Note here that throughout the paper we use the overline on top of a quantity to emphasize that it is an unperturbed quantity by the beam self-fields. In an accelerator with typical magnetic elements, including dipoles, quadrupoles, and sextupoles, $A_{s}$ can be expressed as

$$
\begin{aligned}
A_{s}= & -B_{y}(s) x\left(1-\frac{x}{2 \rho_{x}}\right)-B_{x}(s) y\left(1-\frac{y}{2 \rho_{y}}\right) \\
& +\frac{G_{n}(s)}{2}\left(y^{2}-x^{2}\right)+G_{s}(s) x y+S(s)\left(\frac{1}{2} x y^{2}-\frac{1}{6} x^{3}\right)
\end{aligned}
$$


where the first two terms correspond to dipole components, the third and fourth terms correspond to the normal and skew quadrupole components respectively, where $G_{n}$ and $G_{s}$ are the quadrupole gradient strengths, and the last term acts as sextupole components with $S$ as the sextupole strength.

Substituting Eq. (4) into Eq. (3) leads to the specific expression of the Hamiltonian for our study. The resultant Hamiltonian can be separated into linear and nonlinear parts,

$$
\bar{H}=\bar{H}_{\text {linear }}+\bar{H}_{\text {nonlinear }}
$$

The linear part $\bar{H}_{\text {linear }}$ is

$$
\begin{aligned}
\bar{H}_{\text {linear }}= & \frac{1}{2}\left(K_{x} x^{2}+x^{\prime 2}+K_{y} y^{2}+y^{\prime 2}\right. \\
& \left.+2 G_{s} x y-2 \frac{x \delta}{\rho_{x}}-2 \frac{y \delta}{\rho_{y}}\right)
\end{aligned}
$$

where $K_{x}$ and $K_{y}$ represent the effective focusing. For separate dipole and (horizontal focusing) quadrupole, $K_{x}=\rho_{x}^{-2}+G_{n}$ and $K_{y}=\rho_{y}^{-2}-G_{n}$. Here we note that the above formulation can be applicable to the cases with focusing in combined-function dipoles and with tilt of quadrupoles. The remainder, of which the discussion is beyond the scope of this paper, belongs to the nonlinear part,

$$
\bar{H}_{\text {nonlinear }}=-\frac{S}{B_{y} \rho_{x}}\left(\frac{1}{2} x y^{2}-\frac{1}{6} x^{3}\right)+\cdots
$$

With the given unperturbed Hamiltonian, the singleparticle motion can be obtained by solving the Hamilton's equation of motion,

$$
\frac{d \mathbf{X}}{d s}=J \frac{\partial \bar{H}}{\partial \mathbf{X}}
$$

with $J$ the symplectic matrix

$$
J=\left(\begin{array}{cccccc}
0 & 1 & 0 & 0 & 0 & 0 \\
-1 & 0 & 0 & 0 & 0 & 0 \\
0 & 0 & 0 & 1 & 0 & 0 \\
0 & 0 & -1 & 0 & 0 & 0 \\
0 & 0 & 0 & 0 & 0 & 1 \\
0 & 0 & 0 & 0 & -1 & 0
\end{array}\right) .
$$

The general solution of $\mathbf{X}$ can be expressed as $\mathbf{X}(s)=\circ \mathbf{X}(0)$, where $\mathcal{M}$ is a general map and $\circ$ represents the composition operator. To first order, the solution $\mathbf{X}(s)$ can be written in matrix form as

$$
\mathbf{X}(s)=\mathbf{R} \mathbf{X}(0)
$$

where the six-by-six transport matrix $\mathbf{R}$ is

$$
\mathbf{R}=\left(\begin{array}{cccccc}
R_{11} & R_{12} & R_{13} & R_{14} & 0 & R_{16} \\
R_{21} & R_{22} & R_{23} & R_{24} & 0 & R_{26} \\
R_{31} & R_{32} & R_{33} & R_{34} & 0 & R_{36} \\
R_{41} & R_{42} & R_{43} & R_{44} & 0 & R_{46} \\
R_{51} & R_{52} & R_{53} & R_{54} & 1 & R_{56} \\
0 & 0 & 0 & 0 & 0 & 1
\end{array}\right)
$$

For a collection of particles, we use the six-dimensional phase-space distribution function $f(\mathbf{X} ; s)$ to represent the beam. In many cases, the beam phase space distribution function is assumed to depend on the phase space coordinates through some quadratic form [28], e.g. a Gaussian distribution in one dimension $f(x)=\left(\sqrt{2 \pi} \sigma_{x}\right)^{-1} e^{-x^{2} / 2 \sigma_{x}^{2}}$. Disregarding the static offset of the beam core, or defining the coordinate with respect to this offset, we have $\langle\mathbf{X}(s)\rangle=\mathbf{0}$, where $\langle\ldots\rangle$ takes the average on the beam, $\langle\ldots\rangle \equiv \int(\ldots) f(\mathbf{X}) d \mathbf{X} / \int f(\mathbf{X}) d \mathbf{X}$. Thus it is convenient to characterize a beam using the sigma matrix, defined as

$\begin{aligned} \Sigma(s) & =\left(\begin{array}{cccccc}\langle x x\rangle & \left\langle x x^{\prime}\right\rangle & \langle x y\rangle & \langle x y \prime\rangle & \langle x z\rangle & \langle x \delta\rangle \\ \left\langle x^{\prime} x\right\rangle & \left\langle x^{\prime} x^{\prime}\right\rangle & \left\langle x^{\prime} y\right\rangle & \left\langle x^{\prime} y^{\prime}\right\rangle & \left\langle x^{\prime} z\right\rangle & \left\langle x^{\prime} \delta\right\rangle \\ \langle y x\rangle & \left\langle y x^{\prime}\right\rangle & \langle y y\rangle & \left\langle y y^{\prime}\right\rangle & \langle y z\rangle & \langle y \delta\rangle \\ \left\langle y^{\prime} x\right\rangle & \left\langle y^{\prime} x^{\prime}\right\rangle & \left\langle y^{\prime} y\right\rangle & \left\langle y^{\prime} y^{\prime}\right\rangle & \left\langle y^{\prime} z\right\rangle & \left\langle y^{\prime} \delta\right\rangle \\ \langle z x\rangle & \left\langle z x^{\prime}\right\rangle & \langle z y\rangle & \left\langle z y^{\prime}\right\rangle & \langle z z\rangle & \langle z \delta\rangle \\ \langle\delta x\rangle & \left\langle\delta x^{\prime}\right\rangle & \langle\delta y\rangle & \left\langle\delta y^{\prime}\right\rangle & \langle\delta z\rangle & \langle\delta \delta\rangle\end{array}\right) \\ = & \left\langle\mathbf{X X}^{T}\right\rangle\end{aligned}$

where $\langle x x\rangle \equiv \sigma_{x}^{2},\left\langle x x^{\prime}\right\rangle \equiv \sigma_{x x^{\prime}}$, etc. While we apply the sigma matrix to a specific beam distribution, i.e. Gaussian, we note that Eq. (12) is generally applicable to any type of beam distribution function. Below we shall use the notations $\Sigma_{2 D}=\left\langle\mathbf{X}_{2 D} \mathbf{X}_{2 D}^{T}\right\rangle$ and $\Sigma_{4 D}=\left\langle\mathbf{X}_{4 D} \mathbf{X}_{4 D}^{T}\right\rangle$ to specify the transverse two-by-two and four-by-four subsets of the sigma matrix. The (rms) beam emittance can be determined by $\varepsilon_{6 D}=\sqrt[6]{\operatorname{det} \Sigma}$ for the six-dimensional case and $\varepsilon_{4 D}=\sqrt[4]{\operatorname{det} \Sigma_{4 D}}$ and $\varepsilon_{2 D}=\sqrt{\operatorname{det} \Sigma_{2 D}}$ for fourand two-dimensional cases, respectively. Using the facts that $\mathbf{X}^{T}(0) \Sigma^{-1}(0) \mathbf{X}(0)$ is invariant [28] and Eq. (10), the transport of beam sigma matrix can be formulated as 


$$
\Sigma(s)=\mathbf{R} \Sigma(0) \mathbf{R}^{T}
$$

Since the beam sigma matrix is real and symmetric, it is diagonalizable. That is to say, there always exists a real $\mathbf{V}$ such that

$$
\mathbf{V} \Sigma \mathbf{V}^{T}=\mathbf{D}
$$

with $\operatorname{det}(\mathbf{V})=1$. In Eq. (14), the matrix $\mathbf{V}$ embodies the eigenvectors and the diagonal matrix $\mathbf{D}$ accommodates the corresponding eigenvalues. After the diagonalization, the basis coordinate $\mathbf{X}$ experiences a coordinate transformation to $\mathbf{U}$ with $\mathbf{U}=\mathbf{V X}$. It is obvious that this transformation preserves the invariant (physically, the beam emittance is unchanged through the transformation). Thus we have

$$
\mathbf{X}^{T} \Sigma^{-1} \mathbf{X}=\mathbf{U}^{T} \mathbf{D}^{-1} \mathbf{U}
$$

The above mathematical treatment is in fact equivalent to finding a normal form from a set of coordinates. For example, for two-dimensional subspace $\left(x, x^{\prime}\right)$, throughout the coordinate transformation of Eq. (16) below,

$$
\mathbf{V}_{2 D}=\left(\begin{array}{cc}
\frac{1}{\sqrt{\beta_{x}}} & 0 \\
\frac{\alpha_{x}}{\sqrt{\beta_{x}}} & \sqrt{\beta_{x}}
\end{array}\right)
$$

the invariant $\mathbf{X}_{2 D}^{T} \Sigma_{2 D}^{-1} \mathbf{X}_{2 D}=\varepsilon_{x}^{-1}\left(\gamma_{x} x^{2}+2 \alpha_{x} x x^{\prime}+\beta_{x} x^{\prime 2}\right)$ can be rewritten as $\varepsilon_{x}^{-1}\left(\bar{x}^{2}+\bar{x}^{\prime 2}\right)$ with $\bar{x}=x / \sqrt{\beta_{x}}$ and $\bar{x}^{\prime}=\left(\alpha_{x} x+\beta_{x} x^{\prime}\right) / \sqrt{\beta_{x}}$. Here $\beta_{x}$ and $\alpha_{x}$ are Twiss [29] (or Courant-Snyder [30]) parameters and the 2D beam sigma matrix is parametrized by

$$
\Sigma_{2 D}=\varepsilon_{x}\left(\begin{array}{cc}
\beta_{x} & -\alpha_{x} \\
-\alpha_{x} & \gamma_{x}
\end{array}\right)
$$

where $\varepsilon_{x}$ is the (rms) horizontal geometric emittance of the beam and $\gamma_{x}=\left(1+\alpha_{x}^{2}\right) / \beta_{x}$.

For the subspace $\left(x, x^{\prime}, z, \delta\right)$, we have similarly the transformation given by Eq. (18) [31],

$$
\mathbf{V}_{4 D}=\left(\begin{array}{cccc}
\frac{1}{\sqrt{\beta_{x}}} & 0 & 0 & -\frac{\eta_{x}}{\sqrt{\beta_{x}}} \\
\frac{\alpha_{x}}{\sqrt{\beta_{x}}} & \sqrt{\beta_{x}} & 0 & -\frac{\alpha_{x} \eta_{x}+\beta_{x} \eta_{x}^{\prime}}{\sqrt{\beta_{x}}} \\
\eta_{x}^{\prime} & -\eta_{x} & 1 & 0 \\
0 & 0 & 0 & 1
\end{array}\right)
$$

where $\eta_{x}$ and $\eta_{x}^{\prime}$ are the dispersion function and its derivative, or $R_{16}$ and $R_{26}$ in Eq. (11). In Eqs. (16) and (18), the Twiss (or Courant-Snyder) parameters are assumed to be of the beam's. If a beam is said matched to a beam line, these parameters are equal to the Twiss functions at the entrance of the beam line.

In what follows, we shall use the results obtained in Eqs. (10)-(15) for the development of the Vlasov formalism to describe the evolution of the beam phase space distribution along a beam line.

\section{VLASOV TREATMENT AND ITS LINEARIZATION}

In this section, starting from the fundamental equations, Eq. (8) and the Vlasov equation [32], we will derive the governing equations for microbunching development in a beam line. For a collection of particles, the phase-space distribution function $f(\mathbf{X} ; s)$ is often employed to describe the beam behavior. If the collision between particles is ignored, the evolution can be formulated by the Vlasov equation in the six-dimensional phase space coordinate system,

$$
\frac{\partial f}{\partial s}+\left(\frac{\partial f}{\partial \mathbf{X}}\right)^{T} J \frac{\partial H}{\partial \mathbf{X}}=0
$$

where $H=\bar{H}+H_{1}$ is the total Hamiltonian of the system. Here the unperturbed Hamiltonian $\bar{H}$, as given in Eq. (5), is the pure-optics part (i.e. in the absence of beam self-fields), and $H_{1}$ accounts for the collective interactions in the beam.

For the pure optics case $\left(H_{1}=0\right)$, the unperturbed or equilibrium solution $f(\mathbf{X} ; s)$ can be totally determined by its initial condition, i.e.

$f=\bar{f}(\mathbf{X}(s) ; s)=\bar{f}\left(\mathcal{M}^{-1} \circ \mathbf{X}(s) ; 0\right)=\bar{f}(\mathbf{X}(0) ; 0) \equiv \bar{f}_{0}\left(\mathbf{X}_{0}\right)$

where, for the special case of linear beam transport $\bar{H}=\bar{H}_{\text {linear }}$, from Eq. (10), it follows

$$
\bar{f}\left(\mathcal{M}^{-1} \circ \mathbf{X}(s) ; 0\right)=\bar{f}\left(R^{-1}(s) \mathbf{X}(s) ; 0\right) .
$$

This approach to solving the first-order partial differential equation is known as the method of characteristics.

To proceed, if we are interested in the onset of beam instability, we can assume, to first order, $f=\bar{f}+f_{1}$, where $f_{1} \ll \bar{f}$. Then we substitute $f=\bar{f}+f_{1}$ into Eq. (19) and retain the terms up to first order. We have the equations for zeroth order,

$$
\frac{\partial \bar{f}}{\partial s}+\left(\frac{\partial \bar{f}}{\partial \mathbf{X}}\right)^{T} J \frac{\partial \bar{H}}{\partial \mathbf{X}}=0
$$

and, for the first order, 


$$
\frac{\partial f_{1}}{\partial s}+\left(\frac{\partial f_{1}}{\partial \mathbf{X}}\right)^{T} J \frac{\partial \bar{H}}{\partial \mathbf{X}}-\frac{\partial H_{1}}{\partial z} \frac{\partial \bar{f}}{\partial \delta}=0
$$

To our interest we restrict our study to the case where the collective energy kick is only in the longitudinal direction. From Hamilton's equation, $\partial H_{1} / \partial z=-d \delta / d s$. The energy kick due to the collective force at $s=\tau$ can be explicitly expressed as

$$
\frac{d \delta}{d \tau}=-\frac{N r_{e}}{\gamma} \int \frac{d \kappa}{2 \pi} Z_{0}^{\|}(\kappa ; \tau) b(\kappa ; \tau) e^{i \kappa z_{\tau}}
$$

where $N$ is the total number of particles in a beam, $r_{e}$ the classical radius of the electron, $\gamma \equiv E / m_{e} c^{2}$ the electron relativistic factor, $Z_{0}^{\|}$the impedance per unit length, $\kappa$, as a dummy variable, representing the spatial frequency contents of the beam, and $b(\kappa ; \tau)$ is the density modulation or bunching factor, defined as

$$
b\left(k_{z} ; s\right)=\frac{1}{N} \int d \mathbf{X} e^{-i k_{z} z_{s}} f_{1}(\mathbf{X} ; s)
$$

where $k_{z}$ is the spatial modulation frequency and $\lambda=2 \pi / k_{z}$ can be considered as the modulation wavelength. A discussion is deserved here regarding an assumption made behind Eq. (25). In Eq. (25) the coasting beam approximation has been made, i.e. $\int d \mathbf{X} e^{-i k_{z} z_{s}} \bar{f}(\mathbf{X} ; s)=0$ for nonvanishing $k_{z}$. In many situations, where the characteristic length of the microbunched structure atop the unperturbed distribution is small compared with the full bunch duration, Eq. (25) can properly characterize the microbunching. The coasting beam approximation here is to be compared with the bunched-beam model. In the case when a microbunched structure is comparable to the overall bunch duration, the spectra of the bulk- and microbunches can interfere. Then coupling between different spatial frequencies $k_{z}$ via the finite-bunch length comes into play. When it comes to critical bunch compression or parasitic compression [33], the bunched-beam model has to be considered.

Having integrated Eq. (23) over $s$ along the unperturbed phase space trajectory and taking advantage of the total derivative, we have the perturbed phase space distribution function after the energy kick

$$
f_{1}\left(\mathbf{X}_{s}\right) \simeq f_{1}^{(0)}\left(\mathbf{X}_{0}\right)-\int_{0}^{s} d \tau \frac{\partial \bar{f}\left(\mathbf{X}_{\tau}\right)}{\partial \delta} \frac{d \delta}{d \tau}
$$

where $f_{1}^{(0)}\left(\mathbf{X}_{0}\right)$, being the small perturbed distribution function, is evaluated before the energy kick. This expression, considered as a form of the linearized Vlasov equation [4], will be used below and referred to as a fundamental relation. To proceed, we first need to specify the initial unperturbed electron phase space distribution. We assume the Gaussian distribution in the six-dimensional phase space coordinate,

$$
\bar{f}_{0}\left(\mathbf{X}_{0}\right)=\frac{N}{(2 \pi)^{3} \sqrt{\operatorname{det} \Sigma_{0}}} \exp \left\{\frac{-1}{2} \mathbf{X}_{0}^{T} \Sigma_{0}^{-1} \mathbf{X}_{0}\right\}
$$

where we remind the reader that the overline atop $f_{0}$ is used to denote the unperturbed distribution, and the subscript "0" stands for the quantity evaluated at $s=0$. For an uncoupled beam, the beam distribution can be parametrized by the Twiss (or Courant-Snyder) parameters, as expressed in Eq. (17). The invariant for $\left(x, x^{\prime}\right)$ is

$$
\mathbf{X}_{2 D, 0}^{T} \Sigma_{2 D}^{-1} \mathbf{X}_{2 D, 0}=\frac{x_{0}^{2}+\left(\beta_{x 0} x_{0}{ }^{\prime}+\alpha_{x 0} x_{0}\right)^{2}}{\varepsilon_{x 0} \beta_{x 0}}
$$

with $\operatorname{det}\left(\Sigma_{2 D}\right)=\varepsilon_{x 0}^{2}$. The invariant for $\left(y, y^{\prime}\right)$ is similar to Eq. (28) and thus not shown here. Equation (27) becomes

$$
\begin{aligned}
\bar{f}_{0}\left(\mathbf{X}_{0}\right)= & \frac{N}{(2 \pi)^{3} \varepsilon_{x 0} \varepsilon_{y 0} \sigma_{z 0} \sigma_{\delta 0}} \\
& \times e^{-\frac{x_{0}^{2}+\left(\beta_{x} x_{0}^{\prime}+\alpha_{x} x_{0}\right)^{2}}{2 \varepsilon_{x 0} \beta_{x 0}}-\frac{\hat{y}_{0}^{2}+\left(\beta_{y 0} y_{0}^{\prime}+\alpha_{y 0} y_{0}\right)^{2}}{2 \varepsilon_{y 0} \beta_{y 0}}-\frac{z_{0}^{2}}{2 \sigma_{z 0}^{2}}-\frac{\left(\delta_{0}^{2}-2 h z_{0} \delta_{0}\right)}{2 \sigma_{\delta 0}^{2}}}
\end{aligned}
$$

Here $\varepsilon_{x 0}$ and $\varepsilon_{y 0}$ are the rms horizontal and vertical geometric emittances, respectively. $\alpha_{x 0}, \alpha_{y 0}, \beta_{x 0}$ and $\beta_{y 0}$ are the initial Twiss (or Courant-Snyder) parameters, $\sigma_{z 0}$ for the rms bunch length, $\sigma_{\delta 0}$ for the rms uncorrelated (or, slice) relative energy spread, and $h=\langle z \delta\rangle / \sigma_{z 0}^{2}$ for the linear chirp parameter. When the coasting beam approximation is made, Eq. (29) becomes

$$
\begin{aligned}
\bar{f}_{0}\left(\mathbf{X}_{0}\right) \simeq & \frac{n_{0}}{(2 \pi)^{3} \varepsilon_{x 0} \varepsilon_{y 0} \sigma_{\delta 0}} \\
& \times e^{-\frac{x_{0}^{2}+\left(\beta_{x x} x_{0}^{\prime}+\alpha_{x 0} x_{0}\right)^{2}}{2 \varepsilon_{x 0} \beta_{x 0}}-\frac{y_{0}^{2}+\left(\beta_{y 0} y_{0}^{\prime}+\alpha_{y 0} y_{0}\right)^{2}}{2 \varepsilon_{y 0} \beta_{y 0}}-\frac{\left(\delta_{0}-h z_{0}\right)^{2}}{2 \sigma_{\delta 0}}}
\end{aligned}
$$

where $n_{0} \simeq N / \sigma_{z 0}$ is the number density of the bunch.

Since we are interested in the MBI along a beam line, microbunching can be quantified by the Fourier transformation of the $z$ coordinate of the perturbed phase space distribution function as the density modulation (or, bunching factor), i.e. Eq. (25). In addition, microbunching can arise from or result in energy modulation, which is quantified by the Fourier transformation of the energymodulated phase space distribution,

$$
p\left(k_{z} ; s\right)=\frac{1}{N} \int d \mathbf{X} e^{-i k_{z} z_{s}}(\delta-h z) f_{1}(\mathbf{X} ; s) .
$$

Note that, in Eqs. (25) and (31), the phase space variables are evaluated at $s$. For $s=0$, we quantify the initial density and energy modulations as follows: 


$$
b_{0}\left(k_{0}\right)=b\left(k_{z} ; 0\right)=\frac{n_{0}}{N} \int d z_{0}\left(\frac{\Delta n\left(z_{0}\right)}{n_{0}}\right) e^{-i k_{0} z_{0}}
$$

and

$$
p_{0}\left(k_{0}\right)=p\left(k_{z} ; 0\right)=\frac{n_{0}}{N} \int d z_{0} \Delta \delta\left(z_{0}\right) e^{-i k_{0} z_{0}}
$$

where $\Delta \delta\left(z_{0}\right)=\delta_{0}-h z_{0}$.

Having discussed all the necessary information, our next goal is to derive the governing equations for $b\left(k_{z} ; s\right)$ and $p\left(k_{z} ; s\right)$. By multiplying on both sides of Eq. (26) with $N^{-1} \exp \left(-i k_{z}(s) z_{s}\right)$, and integrating over the sixdimensional phase space $\mathbf{X}$, we have

$$
\begin{aligned}
b\left(k_{z} ; s\right)= & b^{(0)}\left(k_{z} ; s\right)+\frac{1}{N} \int_{0}^{s} d \tau i k_{z}(s) \frac{I_{b}(\tau)}{\gamma I_{A}} R_{56}(\tau \rightarrow s) \\
& \times \int \frac{d \kappa}{2 \pi} Z_{0}^{\|}(\kappa ; \tau) b(\kappa ; \tau) \int d \mathbf{X}_{\tau} \bar{f}\left(\mathbf{X}_{\tau}\right) e^{-i k_{z} z_{s}\left(\mathbf{X}_{\tau}\right)+i \kappa z_{\tau}}
\end{aligned}
$$

where $I_{b}(\tau)=e c n_{0} / C(\tau)$ is the instantaneous bunch current at $s=\tau$ with $C(\tau)=\left[1-h R_{56}(\tau)\right]^{-1}$ the bunch compression factor, and $I_{A}$ is the Alfven current $(\sim 17 \mathrm{kA}) . \quad R_{56}(\tau \rightarrow s)=\left[\mathbf{R}(s) \mathbf{R}^{-1}(\tau)\right]_{56}$ is the relative momentum compaction function from $\tau$ to $s$. The first term on the right-hand side of Eq. (34) is evaluated by Eq. (25) using $f_{1}(\mathbf{X} ; s)=f_{1}^{(0)}\left(\mathbf{X}_{s}\right)$ [an explicit form of $f_{1}^{(0)}\left(\mathbf{X}_{s}\right)$ shall be specified later in either Eq. (48) or (49)]. This term corresponds to the bunching evolution in the absence of collective effects. We want to work out the second term on the right-hand side of Eq. (34) by tracing the relevant phase space coordinates at $s=\tau$ back to the initial location $(s=0)$ with the help of Eqs. (10) and (20). $z_{s}$ in the exponent of the second term on the right-hand side of Eq. (34) can be expressed as

$$
z_{s}=\sum_{j=1}^{6} R_{5 j}(\tau \rightarrow s) X_{\tau}^{j}=\sum_{j=1}^{6} R_{5 j}(s) X_{0}^{j}
$$

where the superscript $j$ indicates the $j$ th component of the phase space coordinate vector $\mathbf{X}$. Similarly, for $z_{\tau}$, we have $z_{\tau}=\sum_{j=1}^{6} R_{5 j}(\tau) X_{0}^{j}$.

Now, in the second term of the right-hand side of Eq. (34), the integration over $\mathbf{X}_{0}$ (originally over $\mathbf{X}_{\tau}$ ) involves the term in the exponent,

$$
\begin{array}{r}
-\frac{1}{2} \mathbf{X}_{0}^{T} \Sigma_{0}^{-1} \mathbf{X}_{0}-i k_{z} z_{s}\left(\mathbf{X}_{0}\right)+i \kappa z_{\tau}\left(\mathbf{X}_{0}\right) \\
=-\frac{1}{2} \mathbf{X}_{0}^{T} \Sigma_{0}^{-1} \mathbf{X}_{0}-i \sum_{j=1}^{6} \Re_{5 j}(s, \tau) X_{0}^{j}
\end{array}
$$

where we have defined

$$
\begin{aligned}
\Re_{5 j}(s, \tau) & =k_{z}(s) R_{5 j}(s)-\kappa(\tau) R_{5 j}(\tau), \\
j & =1,2, \ldots, 6 .
\end{aligned}
$$

For a coupled beam, the term $\mathbf{X}_{0}^{T} \Sigma_{0}^{-1} \mathbf{X}_{0}$ in Eq. (36) usually consists of the mixed terms, such as $x x^{\prime}, x y, x y^{\prime}$, etc. These mixed terms can very much complicate the integration. As discussed in Sec. II [see Eq. (15)], the term $\mathbf{X}_{0}^{T} \Sigma_{0}^{-1} \mathbf{X}_{0}$ can be always diagonalized. We are particularly interested in the case of transversely coupled beams, i.e. $\mathbf{X}_{0}^{T} \Sigma_{0}^{-1} \mathbf{X}_{0}=\mathbf{X}_{4 D, 0}^{T} \Sigma_{4 D, 0}^{-1} \mathbf{X}_{4 D, 0}+\frac{\left(\delta_{0}-h z_{0}\right)^{2}}{\sigma_{\delta 0}^{2}}$, where $\mathbf{X}_{4 D, 0}=$ $\left(x_{0}, x_{0}^{\prime}, y_{0}, y_{0}^{\prime}\right)^{T}$. Following Eqs. (14) and (15), the integration over $\mathbf{X}_{4 D, 0}$ now becomes that over $\mathbf{U}_{4 D}=\left(u_{1}, u_{2}, u_{3}, u_{4}\right)^{T}$, and we have

$$
\begin{gathered}
\int d \mathbf{X}_{4 D, 0} \exp \left\{-\frac{1}{2} \mathbf{X}_{4 D, 0}^{T} \Sigma_{4 D, 0}^{-1} \mathbf{X}_{4 D, 0}-i \sum_{j=1}^{4} \Re_{5 j}(s, \tau) X_{j, 0}\right\} \\
=\prod_{m=1}^{4} \int d u_{m} \exp \left\{-\frac{1}{2} D_{m m}^{-1} u_{m}^{2}-i \sum_{j=1}^{4} \Re_{5 j}(s, \tau) \mathbf{V}_{j m}^{-1} u_{m}\right\} \\
=\prod_{m=1}^{4} \sqrt{\frac{2 \pi}{D_{m m}^{-1}}} \exp \left\{-\frac{1}{2 D_{m m}^{-1}}\left[\sum_{j=1}^{4} \Re_{5 j}(s, \tau) \mathbf{V}_{j m}^{-1}\right]^{2}\right\}
\end{gathered}
$$

where $D_{m m}^{-1}$ for $m=1$ to 4 are diagonal terms of $\mathbf{D}^{-1}$. The positive definiteness of $\mathbf{X}_{4 D, 0}^{T} \Sigma_{4 D, 0}^{-1} \mathbf{X}_{4 D, 0}$ assures that of $\mathbf{U}_{4 D}^{T} \mathbf{D}^{-1} \mathbf{U}_{4 D}$, and thus $D_{m m}^{-1}>0$. From Eqs. (34) and (38), we have

$$
\begin{aligned}
& \int \frac{d \kappa}{2 \pi} Z_{0}^{\|}(\kappa ; \tau) b(\kappa ; \tau) \int d \mathbf{X}_{0} \bar{f}_{0}\left(\mathbf{X}_{0}\right) e^{-i k_{z} z_{s}\left(\mathbf{X}_{0}\right)+i \kappa z_{\tau}\left(\mathbf{X}_{0}\right)} \\
& \quad=Z_{0}^{\|}\left(k_{z}(\tau) ; \tau\right) b\left(k_{z}(\tau) ; \tau\right)\{L . D . ; s, \tau\}
\end{aligned}
$$

where the term $\{L . D . ; s, \tau\}$ characterizes the smearing effect or Landau damping,

$$
\begin{aligned}
\{L . D . ; s, \tau\}= & \exp \left\{-\frac{1}{2} \sum_{m=1}^{4} \frac{1}{D_{m m}^{-1}}\left(\sum_{j=1}^{4} \Re_{5 j}(s, \tau) V_{j m}^{-1}\right)^{2}\right. \\
& \left.-\frac{1}{2} \sigma_{\delta 0}^{2} \Re_{56}(s, \tau)\right\}
\end{aligned}
$$

and

$$
\begin{aligned}
\Re_{5 j}(s, \tau) & =k_{z}(s) R_{5 j}(s)-k_{z}(\tau) R_{5 j}(\tau) \\
& =k_{0}\left[C(s) R_{5 j}(s)-C(\tau) R_{5 j}(\tau)\right], \\
j & =1,2,3,4,6
\end{aligned}
$$


where use has been made of the coasting beam approximation. Note that in Eq. (39), $\int \frac{d \kappa}{2 \pi} \int d z_{0} \exp \left\{-i\left[k_{z}(s) /\right.\right.$ $\left.C(s)-\kappa(\tau) / C(\tau)] z_{0}\right\}=1$ when $\kappa(\tau)=k_{z}(s) C(\tau) / C(s)$ and $C(\tau)$ is the bunch compression factor at $s=\tau$.
Note that the matrix $\mathbf{V}$ in Eq. (40) has been obtained when we diagonalize $\Sigma_{0}$ (or $\Sigma_{0}^{-1}$ ) [see Eq. (14)]. For the case of an uncoupled beam, Eq. (40) is reduced to [4]

$$
\{L . D . ; s, \tau\}=\exp \left\{\frac{-1}{2}\left[\begin{array}{c}
\varepsilon_{x 0} \beta_{x 0}\left(\Re_{51}(s, \tau)-\frac{\alpha_{x 0}}{\beta_{x 0}} \Re_{52}(s, \tau)\right)^{2}+\frac{\varepsilon_{x 0}}{\beta_{x 0}} \Re_{52}^{2}(s, \tau)+ \\
\varepsilon_{y 0} \beta_{y 0}\left(\Re_{53}(s, \tau)-\frac{\alpha_{y 0}}{\beta_{y 0}} \Re_{54}(s, \tau)\right)^{2}+\frac{\varepsilon_{y 0}}{\beta_{y 0}} \Re_{54}^{2}(s, \tau)+\sigma_{\delta 0}^{2} \Re_{56}^{2}(s, \tau)
\end{array}\right]\right\} .
$$

Putting it all together, we arrive at the governing equation, Eq. (34), for density modulation (or bunching factor) as a compact integral equation,

$$
b\left(k_{z} ; s\right)=b^{(0)}\left(k_{z} ; s\right)+\int_{0}^{s} d \tau K(\tau, s) b\left(k_{z} ; \tau\right)
$$

where the kernel function is

$$
K(\tau, s)=i k_{z}(s) \frac{I_{b}(\tau)}{\gamma I_{A}} R_{56}(\tau \rightarrow s) Z_{0}^{\|}\left(k_{z} ; \tau\right)\{L . D . ; s, \tau\}
$$

Similar to the case of density modulation, we can also obtain the equation for energy modulation from the fundamental relation, Eq. (26). Multiplying both sides of Eq. (26) by $\left(\delta_{s}-h z_{s}\right) \times \exp \left(-i k_{z}(s) z_{s}\right)$ and integrating over the six-dimensional phase space $\mathbf{X}$, the resultant governing equation can be expressed in terms of Eqs. (25) and (31),

$p\left(k_{z} ; s\right)=p^{(0)}\left(k_{z} ; s\right)+\int_{0}^{s} d \tau[M(\tau, s)-L(\tau, s)] b\left(k_{z} ; \tau\right)$

where the kernel functions are

$$
\begin{aligned}
M(\tau, s) & =i k_{z}(s) \frac{I_{b}(\tau)}{\gamma I_{A}} \sigma_{\delta 0}^{2} R_{56}(\tau \rightarrow s) \Re_{56}(s, \tau) Z_{0}^{\|}\left(k_{z} ; \tau\right) \\
& =\sigma_{\delta 0}^{2} \Re_{56}(s, \tau) K(\tau, s)
\end{aligned}
$$

and

$$
L(\tau, s)=\frac{I_{b}(\tau)}{\gamma I_{A}} Z_{0}^{\|}\left(k_{z} ; \tau\right)
$$

Now we have derived two integral equations that govern the evolution of both density and energy modulations along a beam line. However we have not yet obtained $b^{(0)}\left(k_{z} ; s\right)$ and $p^{(0)}\left(k_{z} ; s\right)$. This requires the explicit expressions of the initial density- and energy-perturbed phase-space distribution functions. They can be respectively formulated as [see also Eqs. (30), (32) and (33)]

$$
f_{1}^{(0, d)}\left(\mathbf{X}_{0}\right)=\frac{\Delta n\left(z_{0}\right)}{n_{0}} \bar{f}_{0}\left(\mathbf{X}_{0}\right)
$$

and

$$
f_{1}^{(0, e)}\left(\mathbf{X}_{0}\right)=\frac{\left(\delta_{0}-h z_{0}\right) \Delta \delta\left(z_{0}\right)}{\sigma_{\delta 0}^{2}} \bar{f}_{0}\left(\mathbf{X}_{0}\right)
$$

where a second superscript, $(d)$ or $(e)$, is used to denote either the density or energy modulation.

Substituting Eqs. (48) and (49) into Eqs. (25) and (31), we have a total of four combinations,

$$
\begin{gathered}
b^{(0, d)}\left(k_{z} ; s\right)=b_{0}\left(k_{0}\right)\{L . D . ; s, 0\} \\
b^{(0, e)}\left(k_{z} ; s\right)=-i k_{z}(s) R_{56}(s) p_{0}\left(k_{0}\right)\{L . D . ; s, 0\} \\
p^{(0, d)}\left(k_{z} ; s\right)=-i k_{z}(s) R_{56}(s) \sigma_{\delta 0}^{2} b_{0}\left(k_{0}\right)\{L . D . ; s, 0\} \\
p^{(0, e)}\left(k_{z} ; s\right)=\left(1-k_{z}^{2}(s) R_{56}^{2}(s) \sigma_{\delta 0}^{2}\right) p_{0}\left(k_{0}\right)\{L . D . ; s, 0\}
\end{gathered}
$$

which are, respectively, the density modulations due to initial density [Eq. (50)] and energy [Eq. (51)] modulations, and energy modulations due to initial density [Eq. (52)] and energy [Eq. (53)] modulations. Note that Eqs. (50)-(53) determine the pure-optics evolution of density and energy modulations. One can see, in the absence of collective effects, a (downstream) resultant density modulation can be either inherent, i.e. from Eq. (50), or transformed from energy modulation via the momentum compaction function $R_{56}$ upstream a beam line, i.e. Eq. (51). Similarly, for a (downstream) energy modulation, it can be either inherent, Eq. (53), or resulted from finite energy spread in a densitymodulated beam, Eq. (52). In the presence of collective effects, such as CSR and LSC, they will complicate the conversion between density and energy modulations. In total, we have four different combinations, each of which corresponds to an integral equation derived in Eqs. (43)-(47). By dividing a beam line into grids, these four integral equations can be cast into vector/matrix form as 


$$
\left[\begin{array}{c}
\mathbf{b}^{(d)} \\
\mathbf{b}^{(e)} \\
\mathbf{p}^{(d)} \\
\mathbf{p}^{(e)}
\end{array}\right]=\left(\begin{array}{cccc}
(\mathbf{1}-\mathbf{K})^{-1} & \mathbf{0} & \mathbf{0} & \mathbf{0} \\
\mathbf{0} & (\mathbf{1}-\mathbf{K})^{-1} & \mathbf{0} & \mathbf{0} \\
(\mathbf{M}-\mathbf{L})(\mathbf{1}-\mathbf{K})^{-1} & \mathbf{0} & \mathbf{1} & \mathbf{0} \\
\mathbf{0} & (\mathbf{M}-\mathbf{L})(\mathbf{1}-\mathbf{K})^{-1} & \mathbf{0} & \mathbf{1}
\end{array}\right)_{4 M \times 4 M}\left[\begin{array}{c}
\mathbf{b}^{(0, d)} \\
\mathbf{b}_{0}^{(0, e)} \\
\mathbf{p}_{0}^{(0, d)} \\
\mathbf{p}_{0}^{(0, e)}
\end{array}\right]
$$

where $\quad \mathbf{b} \equiv\left[b\left(s_{1}\right), b\left(s_{2}\right), \ldots, b\left(s_{M}\right)\right]^{T} \quad$ and $\quad \mathbf{p} \equiv\left[p\left(s_{1}\right)\right.$, $\left.p\left(s_{2}\right), \ldots, p\left(s_{M}\right)\right]^{T}$, and $s_{i}(i=1,2, \ldots, M)$ represents the grid along a beam line $\left(s_{1}=0\right.$ and $s_{M}=s_{f}$ denote the entrance and the exit of a beam line). $\mathbf{1}$ is the $M$-by- $M$ identity matrix, $\mathbf{0}$ is the zero matrix, and $\mathbf{K}, \mathbf{L}$, and $\mathbf{M}$ are the matrix representations of Eqs. (44), (46), and (47). By virtue of the matrix language, Eq. (43) has been expressed as $\mathbf{b}=\mathbf{b}^{(0)}+\mathbf{K} \mathbf{b}$ and Eq. (45) as $\mathbf{p}=\mathbf{p}^{(0)}+(\mathbf{M}-\mathbf{L}) \mathbf{b}$. The existence of the inverse matrix is assumed. Equation (54) can be expressed in a more compact form as

$$
\left[\begin{array}{l}
\mathbf{b} \\
\mathbf{p}
\end{array}\right]=\left(\begin{array}{cc}
(\mathbf{1}-\mathbf{K})^{-1} & \mathbf{0} \\
(\mathbf{M}-\mathbf{L})(\mathbf{1}-\mathbf{K})^{-1} & \mathbf{1}
\end{array}\right)\left[\begin{array}{l}
\mathbf{b}^{(0)} \\
\mathbf{p}^{(0)}
\end{array}\right]
$$

where $\mathbf{b} \equiv \mathbf{b}^{(d)}+\mathbf{b}^{(e)}$ and $\mathbf{p} \equiv \mathbf{p}^{(d)}+\mathbf{p}^{(e)}$.

The quantity of particular interest is the microbunching gain, defined as the modular ratio of density modulations at a certain location $s$ to the initial location $s=0$,

$$
G(s) \equiv\left|\frac{b\left(k_{z} ; s\right)}{b^{(0)}\left(k_{0} ; 0\right)}\right|
$$

When it comes to different types of modulations, e.g. the density-to-energy or energy-to-density microbunching, we directly refer to values of $b\left(k_{z} ; s\right)$ and $p\left(k_{z} ; s\right)$, evaluated in units of $b^{(0)}\left(k_{0} ; 0\right)$ or $p^{(0)}\left(k_{0} ; 0\right)$.

To facilitate the discussion, in what follows we call $G(s)$, the gain function, which is a function of $s$ for a given modulation wave number, and refer to $G_{f}(\lambda)=G\left(s=s_{f}\right.$; $\left.\lambda=2 \pi / k_{z}\right)$ as the gain spectrum, a function of modulation wavelength at the exit (the subscript " $f$ " indicates the exit of a beam line).

\section{IMPEDANCE MODELS}

In this section we summarize relevant collective effects considered in this paper, which include CSR and LSC effects. For an electron beam traversing a finite-length dipole, CSR can have both steady-state and transient effects. In addition, when a beam goes through a long transport line, LSC can have a significant effect on accumulating energy modulations. Here we quote the relevant impedance expressions in $\operatorname{cgs}$ units without derivation:

\section{A. 1D Free-space CSR}

For a relativistic electron beam $(\beta \rightarrow 1$, but $\gamma<\infty)$ traversing a bending dipole, the 1D free-space steadystate CSR impedance per unit length can be expressed as [34]

$$
\begin{aligned}
& \operatorname{Re}\left[Z_{\mathrm{CSR}}^{\text {s.s.NUR }}\left(k_{z}(s) ; s\right)\right] \\
& =\frac{-2 \pi k_{z}^{1 / 3}(s)}{|\rho(s)|^{2 / 3}} \mathrm{Ai}^{\prime}(x)+\frac{k_{z}(s) \pi}{\gamma^{2}}\left(\int_{0}^{x} \mathrm{Ai}(\varsigma) d \varsigma-\frac{1}{3}\right) \\
& \operatorname{Im}\left[Z_{\mathrm{CSR}}^{\text {s.s.NUR }}\left(k_{z}(s) ; s\right)\right] \\
& \simeq \frac{2 \pi k_{z}^{1 / 3}(s)}{|\rho(s)|^{2 / 3}}\left\{\frac{1}{3} \mathrm{Bi}^{\prime}(x)+\int_{0}^{x}\left[\mathrm{Ai}^{\prime}(x) \operatorname{Bi}(t)-\mathrm{Ai}(t) \mathrm{Bi}^{\prime}(x)\right] d t\right\}
\end{aligned}
$$

where $x=\left(k_{z}(s)|\rho(s)|\right)^{2 / 3} / \gamma^{2}, k_{z}=2 \pi / \lambda$ is the modulation wave number, $\rho(s)$ is the bending radius, and $\mathrm{Ai}$ and $\mathrm{Bi}$ are Airy functions. Under ultrarelativistic approximation $(\beta \rightarrow 1, \gamma \rightarrow \infty)$, Eq. (57) is reduced to the well-known expression $[35,36]$

$$
Z_{\mathrm{CSR}}^{\text {S.S.UR }}\left[k_{z}(s) ; s\right]=\frac{-i k_{z}^{1 / 3}(s) A}{|\rho(s)|^{2 / 3}}
$$

where $A=-2 \pi\left[\mathrm{Bi}^{\prime}(0) / 3+i \mathrm{Ai}^{\prime}(0)\right] \approx-0.94+1.63 i$.

Prior to reaching the steady state, the beam entering a bend from a straight section would experience the so-called entrance transient state, where the impedance per unit length can be obtained by Laplace transformation of the corresponding wake field [37-39]:

$Z_{\mathrm{CSR}}^{\mathrm{ent}}\left[k_{z}(s) ; s\right]=\frac{-4}{s^{*}} e^{-4 i \mu(s)}+\frac{4}{3 s^{*}}[i \mu(s)]^{1 / 3} \Gamma\left(\frac{-1}{3}, i \mu(s)\right)$

where $\mu(s)=k_{z}(s) z_{L}(s), s^{*}$ is the longitudinal coordinate measured from the dipole entrance, $z_{L}=\left(s^{*}\right)^{3} / 24 \rho(s)^{2}$, and $\Gamma$ is the upper incomplete Gamma function.

There are also CSR exit transient effects when a beam exits from a dipole. For the case with CSR fields generated from an upstream electron (at retarded time, traveling along 
the upstream straight section) propagating across the dipole to downstream straight section, the corresponding impedance per unit length can be similarly obtained as Eq. (59) by Laplace transformation of the corresponding wake field (Case C in Ref. [39]):

$$
Z_{\mathrm{CSR}}^{\mathrm{C}}\left[k_{z}(s) ; s\right]=\frac{-4}{L_{b}+2 s^{*}} e^{\frac{-i k_{z}(s) L_{b}^{2}}{6|\rho(s)|^{2}}\left(L_{b}+3 s^{*}\right)}
$$

where $s^{*}$ is the longitudinal coordinate measured from the dipole exit and $L_{b}$ is the dipole length. Other quantities are defined the same as above.

For the impedance expression of the case where CSR fields are generated from an electron (at retarded time) within a dipole propagating downstream the straight section, we adopt the following expression for the CSR drift impedance [40]:

$$
Z_{\mathrm{CSR}}^{\mathrm{drif}}\left[k_{z}(s) ; s\right]=\frac{4}{\rho} \int_{0}^{\theta_{m}} d \vartheta \frac{d f\left(s^{*}, \vartheta\right)}{d \vartheta} e^{-i k_{z}(s) \Delta z\left(s^{*}, \vartheta\right)}
$$

where

$$
f\left(s^{*}, \vartheta\right)=\frac{\frac{2}{\gamma^{2}}\left(\frac{s^{*}}{\rho}+\vartheta\right)+\vartheta^{2}\left(\frac{2 s^{*}}{\rho}+\vartheta\right)}{\frac{4}{\gamma^{2}}\left(\frac{s^{*}}{\rho}+\vartheta\right)^{2}+\vartheta^{2}\left(\frac{2 s^{*}}{\rho}+\vartheta\right)^{2}}
$$

and

$$
\Delta z\left(s^{*}, \vartheta\right)=\frac{s^{*}+\rho \vartheta}{2 \gamma^{2}}+\beta \frac{\rho \vartheta^{3}}{24} \frac{4 s^{*}+\rho \vartheta}{s^{*}+\rho \vartheta}
$$

where $s^{*}$ is again the longitudinal coordinate measured from the exit of the dipole and $\theta_{m}$ is the angle of a bending dipole with radius $\rho . \beta$ and $\gamma$ are relativistic Lorentz factors. Equation (61) is valid for nonultrarelativistic beam energy. The reader is referred to Ref. [40] for the detailed derivation and analysis. Moreover, in the subsequent Vlasov calculation we only include the exit transient effects [Eqs. (60) and (61)] at a nearby upstream bend.

In the above impedance expressions, the impedance depends on the beam line coordinate $s$ and the wave number $k_{z}$. Also the wave number depends again on the beam line coordinate, e.g. if bunch compression is involved, and the impedance is evaluated for a fixed wave number.

\section{B. LSC in Free Space}

Below we adopt the LSC impedance expression derived in Ref. [41]. The beam is assumed transversely uniform with a circular cross section of radius $r_{b}$ [41],

$$
Z_{\mathrm{LSC}}^{\text {on-axis }}\left[k_{z}(s) ; s\right]=\frac{4 i}{\gamma r_{b}(s)} \frac{1-\xi K_{1}(\xi)}{\xi}
$$

where $\xi=k_{z}(s) r_{b}(s) / \gamma$ and $r_{b}(s) \approx \frac{1.747}{2}\left(\sigma_{x}(s)+\sigma_{y}(s)\right)$ [42].

In Eq. (64), the longitudinal field does neither take into account the offset of the bunch centroid nor the transverse dependence of the field along the bunch. We note that this expression is approximate and may not reflect the most general case.

The numerical algorithm of implementing Eq. (54) for density-to-density modulation with inclusion of the above analytical impedance models is detailed in Ref. [43]. The remaining three aspects are similar and we do not repeat them here. The only difference from those described in Ref. [43] is that the initial conditions for the beam are no longer Twiss parameters but the beam sigma matrix $\Sigma_{0}$. Diagonalization of the beam sigma matrix, Eqs. (14)-(15), can be done using standard matrix manipulation tools, e.g. available in matlab.

\section{EXAMPLES}

In this section we apply the results obtained in the previous sections to two example lattices. The first example is the early design of JLEIC CCR [44], and the second one is our recent ERL cooler ring design [45]. The two examples have different beam line designs and distinct natures of the circulating electron beams. For CCR, the beam is nonmagnetized and MBI was not much taken care of in the early design. For the ERL cooler ring, the beam is magnetized and the steering magnets are designed according to Ref. [46]. Presenting the first example here serves two informative purposes. In view of microbunching dynamics, the CCR features an ultrahigh gain, with maximum steady-state CSR gain up to 4000 at $\lambda \approx$ $360 \mu \mathrm{m}$ and even higher when LSC is included, as well as significant longitudinal phase space fragmentation $[6,7]$. In contrast, the arc design of the ERL cooler ring is free from MBI, with maximum gain around unity. From the viewpoint of a sanity test in our newly developed semianalytical Vlasov solver, we use this CCR lattice to confirm that our newly developed solver based on Eq. (54) indeed gives identical results to those obtained by our previous one [43], specialized for nonmagnetized beam transport. The underlying physics of effective suppression of MBI in a magnetized beam transport is then discussed. We also carefully benchmark our semianalytical Vlasov solutions against particle tracking by ELEGANT [26]. Both approaches are found in good agreement.

\section{A. Previous CCR design for nonmagnetized cooling}

The linear optics design was completed as the circulating cooler ring for electron cooling in the ion collider ring at the Medium-energy Electron-Ion Collider (MEIC) project [44] at JLab (now JLEIC [5]). It has been known that maintaining excellent phase space quality for the electron beam is crucial to the electron cooling efficiency. This 
recirculation/decompression

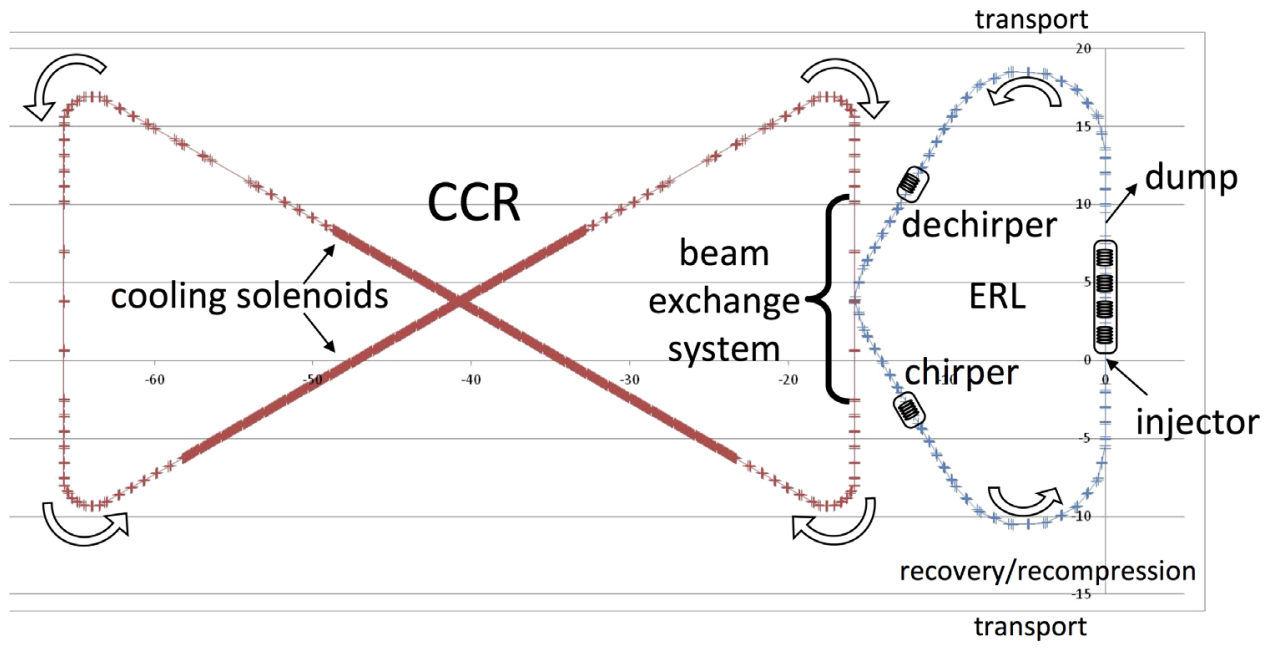

FIG. 1. Schematic layout of the early CCR design for the JLEIC [44].

preliminary design is based upon the topological structure of the figure-8 collider ring, as illustrated in Fig. 1[44]. Such design of an electron cooler ring is characteristic of two 30-m cooling solenoids across the center of the electron collider ring (not shown here) and composed of horizontal dipoles around the four corners and vertical bending dipoles around the two diagonal corners to meet the requirement of vertically stacked figure-8 rings [44]. The circulating electron beam was initially assumed uncoupled in transverse planes. Note that the transverse beam dynamics of horizontal and vertical planes are coupled inside and decoupled outside the cooling solenoids. In the case with a mere CSR effect, which only occurs within bending dipoles, we can artificially take out the solenoid sections in the simulations without affecting the microbunching dynamics. If LSC is to be considered, the two 30-m cooling solenoids should be included.

Table I lists the initial beam and Twiss parameters for the CCR beam line design. The steady-state CSR gain functions $G(s)$ for three different modulation wavelengths are shown in Fig. 2 where we found the microbunching gain with $\lambda=350 \mu \mathrm{m}$ is much larger compared with the other two cases $\lambda=100$ and $1000 \mu \mathrm{m}$, particularly at the last several bends. Because of the frequency dependence of the impedances [see Eqs. (57)-(64)], a more thorough consideration would be to scan a spectral range of modulation wavelengths. Figure 3 shows the steady-state CSR gain spectrum $G_{f}(\lambda)$ as a function of initial modulation wavelengths at the exit of the lattice. In this figure, the same result with two different theoretical formulations is obtained; the red curve, obtained by the formulation derived in Sec. III, and the blue curve, from our previously developed semianalytical Vlasov solver [43] for nonmagnetized beams. This numerically verifies the equivalence of the generalized formulation to the existing one for the special case of transversely uncoupled beams. One can see in Figs. 2 and 3 that the shorter wavelengths enhance the Landau damping or smearing in phase space [through Eq. (40) or (42)] while the longer wavelengths feature negligible CSR effect [see Eqs. (57) and (58)]. Though not shown here, our Vlasov analysis indicates that, with inclusion of CSR transient effects [Eqs. (59)-(63)], the maximum gain can be at least two orders of magnitude larger than the steady-state CSR gain. We notice that with the huge gain shown in Figs. 2 and 3 the microbunching mechanism may enter a nonlinear regime where the linearized Vlasov solutions are no longer valid from a practical point of view. This situation is however beyond the scope of this paper. Here we note that, due to the ultrahigh gain for JLEIC CCR, particle tracking simulation by ELEGANT imposes a significant challenge to obtain convergent results. To compare with the linear theory (or at the onset of $\mathrm{MBI}$ ), the numerically imposed density modulation needs to be small enough to remain in the linear regime while such modulation requires it to be large enough to rise above the numerical noise originated from the limited number of simulation particles. This implies that a large number of simulation particles and long

TABLE I. Initial beam parameters for CCR used in the simulation.

\begin{tabular}{lcc}
\hline \hline Name & Value & Unit \\
\hline Beam energy & 54 & $\mathrm{MeV}$ \\
Bunch charge & 2 & $\mathrm{nC}$ \\
Initial peak bunch current & 60 & $\mathrm{~A}$ \\
Transverse normalized emittance & 3 & $\mu \mathrm{m}$ \\
Compression factor & 1 & \\
Chirp & 0 & $\mathrm{~m}^{-1}$ \\
Energy spread (uncorrelated) & $1.0 \times 10^{-4}$ & \\
\hline \hline
\end{tabular}




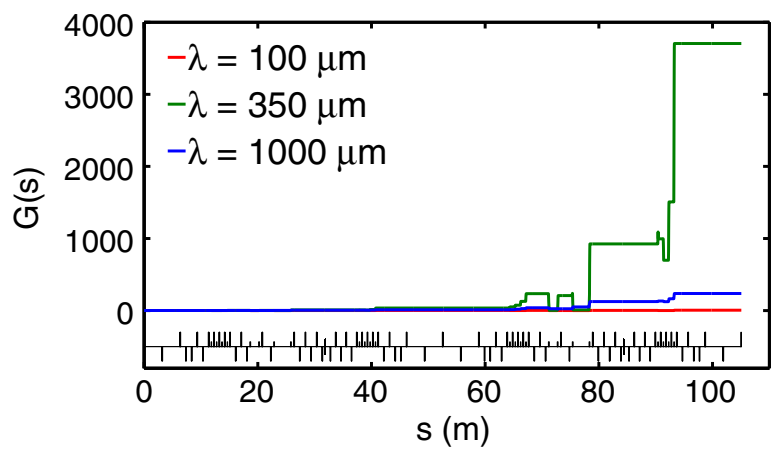

FIG. 2. CSR gain functions $G(s)$ for MEIC CCR lattice: (red) $\lambda=100 \mu \mathrm{m}$, (green) $\lambda=350 \mu \mathrm{m}$, (blue) $\lambda=1000 \mu \mathrm{m}$.

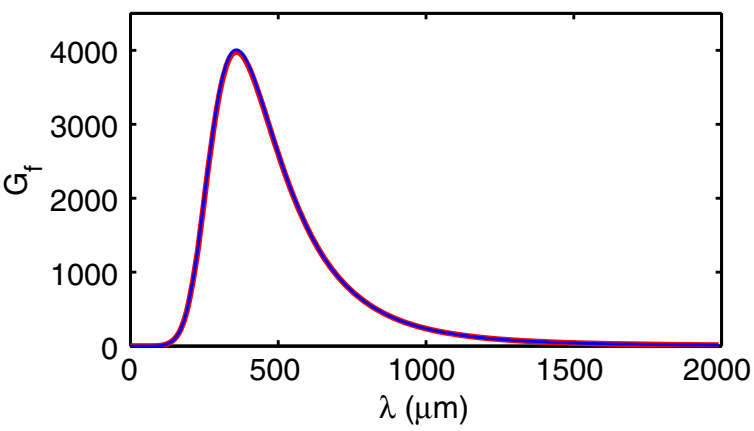

FIG. 3. CSR gain spectra as a function of initial modulation wavelengths for the JLEIC CCR lattice. $G_{f}$ is evaluated as oneturn microbunching gain. The red curve is obtained by the formulation developed in this paper, while the blue curve is from our previously developed semianalytical Vlasov solver [43] for nonmagnetized beams.

computation time are required for reaching convergent results of microbunching gain. Thus, to validate our semianalytical results against ELEGANT, and to ease the numerical difficulties with ELEGANT tracking, we intentionally increase the transverse beam emittances ten times larger than the nominal values shown in Table I (i.e. $\varepsilon_{n x}=\varepsilon_{n y}=30 \mu \mathrm{m}$ ) because larger emittance is known to induce more Landau damping, resulting in lower microbunching gain. Lower gain can thus relieve the stringent requirement of imposing small initial density modulation as well as large number of simulation particles, thus relaxing numerical difficulties. The benchmark results of CCR for the case with $\varepsilon_{n x}=\varepsilon_{n y}=30 \mu \mathrm{m}$ are shown in the Appendix. We remind the reader that in both our semianalytical Vlasov analysis and ELEGANT tracking, we only consider the CSR microbunching instability in a single turn for the CCR. Our study indicates that the preliminary design of CCR for high-energy electron cooling is at risk of microbunching instability; an improved design is required to suppress such instability and/or alternative beam transport schemes should be considered in order to compensate and to circulate the electron beam as many turns as possible while maintaining the high phase space quality of the electron beam required by electron cooling efficiency.

Out investigation shows that, for JLEIC CCR, due to the high bunch charge $(\sim 2 \mathrm{nC})$ and small beam emittance as well as low energy spread $\left(\sim 10^{-4}\right)$, the CSR-induced microbunching quickly accumulates and reaches a maximum gain $\sim 4000$ at $\lambda \approx 360 \mu \mathrm{m}$ in the design. Figure 4 illustrates the longitudinal phase space fragmentation due to CSR using ELEGANT. In the ELEGANT tracking simulation, we use $3 \times 10^{6}$ macroparticles with quiet start. The numerical setting to properly simulate CSR effects within bending dipoles follows that described in Ref. [46]. The number of macroparticles employed here is far from enough to produce the quantitatively converged results. Nevertheless, Fig. 4 serves an informative purpose to qualitatively demonstrate the phase space modulation due to severe CSR effects on microbunching.

\section{B. ERL cooler design for magnetized cooling}

In this subsection we perform the microbunching analyses by considering our recent arc design of the JLEIC ERL cooler ring for magnetized beam cooling. Figure 5 shows the schematic layout of the recent ERL cooler ring design for JLEIC [45]. This design is different from the previous CCR design $[1,44]$, in which the beam was nonmagnetized and the electron beam $(\sim 2 \mathrm{nC}$ with peak current $60 \mathrm{~A})$ was targeted for strong cooling. This current ERL cooler ring design is however to transport magnetized beams and so far designed for weak cooling [21]. A beam with magnetization is generated in the magnetized gun, immersed in a solenoid. Then the beam is injected in the energy-recovered linac and accelerated to about $55 \mathrm{MeV}$. The choice of the
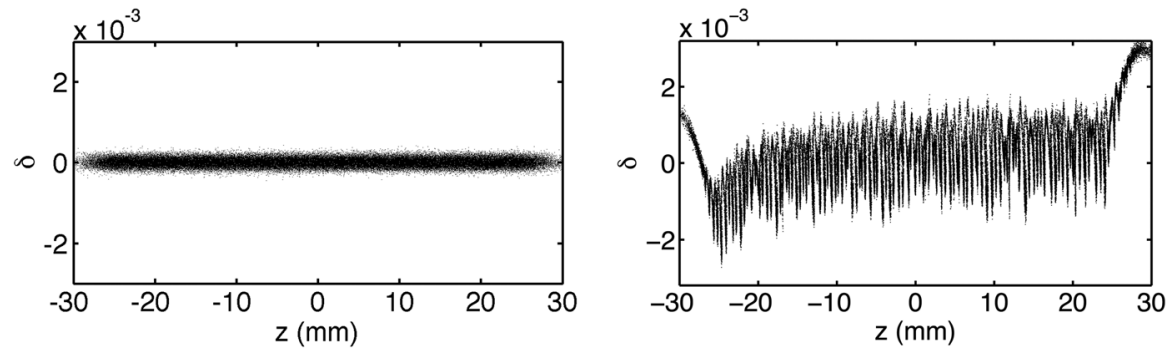

FIG. 4. Longitudinal phase space distributions for the JLEIC CCR: left: initial quiet beam; right: when the beam circulates one turn. Note that $z>0$ is for bunch head. 


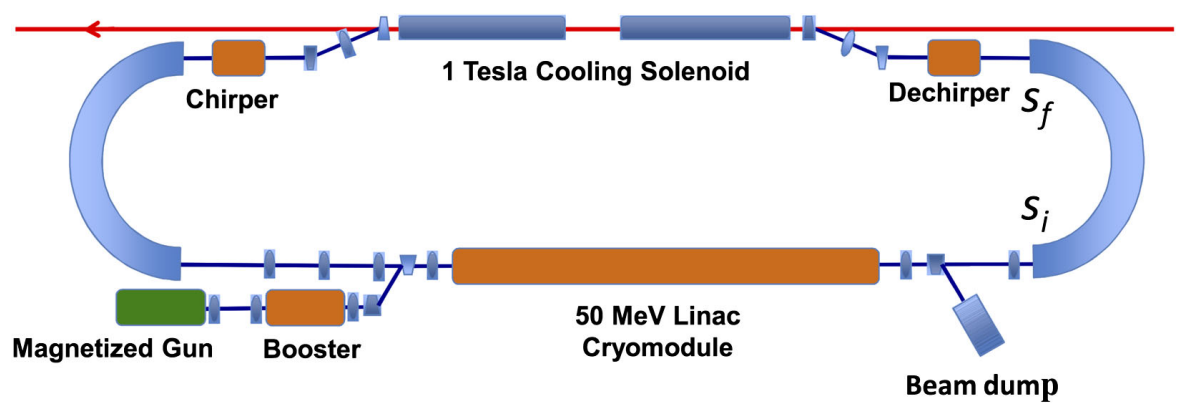

FIG. 5. Schematic layout of the JLEIC ERL cooler design [45].

energy is for the purpose of downstream electron cooling of ion beams. The two arcs, presumed identical, are designed to transport and decompress/compress the beam bunch before/after the cooling section. Table II summarizes the beam parameters at the entrance of the first arc for our simulation. This achromatic arc is composed of eight cells, and each cell is constructed by two inward and one outward bend. The total bending angle is $180^{\circ}$. Each bending dipole is designed as a half-indexed [47] and combined-function dipole. The arc lattice serves to transport the beam, to match toward the downstream solenoid entrance, as well as to preserve the axial symmetry [48].

Figure 6 shows the simulation results for this example arc. An initial nonzero chirp is imposed on the beam so that the bunch is decompressed while it traverses through the arc. The evolution of bunch current is shown in Fig. 6(a). The microbunching gain function, defined in Eq. (56), is illustrated in Fig. 6(b) for $\lambda=300 \mu \mathrm{m}$. In this figure, the dots are obtained from ELEGANT tracking with inclusion of 1D steady-state CSR effect. In ELEGANT, a total of $16 \times 10^{6}$ simulation particles are used and 700 bins are set to ensure the convergence of the results and the minimum resolved modulation wavelength down to $50 \mu \mathrm{m}$. The input beam phase-space distribution for particle tracking is prepared according to Ref. [49]. The data postprocessing follows that described in Ref. [50] and for detailed procedures we refer the reader to Ref. [51]. The overall amplification ratio smaller or around unity indicates that the beam during transport is free from MBI. Figure 6(c) shows the microbunching gain spectra at the exit of the arc as a function of

TABLE II. Initial beam parameters for JLEIC ERL magnetized beam transport.

\begin{tabular}{lcc}
\hline \hline Name & Value & Unit \\
\hline Beam energy & 55 & $\mathrm{MeV}$ \\
Bunch charge & 420 & $\mathrm{pC}$ \\
Initial peak bunch current & 22.5 & $\mathrm{~A}$ \\
4D geometric emittance & 0.11 & $\mu \mathrm{m}$ \\
Compression factor & 0.28 & \\
Chirp & 4.465 & $\mathrm{~m}^{-1}$ \\
Energy spread (uncorrelated) & $1.5 \times 10^{-4}$ & \\
\hline \hline
\end{tabular}

initial modulation wavelength. This figure shows nearly absence of microbunching in the beam transport even with inclusion of both CSR and LSC. From Figs. 6(b) and 6(c), we find both our newly developed semianalytical Vlasov solutions and particle tracking simulations agree with each other. The analysis shows that there is basically no gain growth along the arc. That is, the phase space quality of the beam is well preserved in the transport arc. As a reference, Fig. 6(d) indicates the validity of the 1-D CSR model [36] used in the simulation, where the so-called Derbenev ratio is defined as $\kappa=\sigma_{x} / \lambda^{2 / 3} \rho^{1 / 3}$ [not to be confused with the dummy variable used in Eq. (24)]. This ratio is assumed to be small when the 1D model is valid. When the ratio is no longer small, the transverse variation of the CSR field needs to be taken into account, and a 2D CSR analysis shall be required [52,53].

Compared to a nonmagnetized beam, for example the beam in the JLEIC CCR, a general feature of a magnetized beam is the (much) larger transverse beam size because of its intrinsic angular momentum. This larger beam size can have an effective smearing effect at locations where $R_{51}(s) \sigma_{x}(s)>\lambda(s)$. In this magnetized beam transport arc example, the maximum correlated length $R_{51} \sigma_{x} \approx 2 \mathrm{~mm}$, is much longer than the modulation wavelength of interest. The smearing mechanism is similar to that due to the finite energy spread, which becomes effective when $R_{56}(s) \sigma_{\delta}(s)>\lambda(s)$. In the example of the magnetized ERL cooler arc design, it is found that the effect of smearing in the longitudinal plane, $R_{56} \sigma_{\delta} \approx 80 \mu \mathrm{m}$, is negligible, compared with several to tens $\mu \mathrm{m}$ [Fig. 6(c)]. Therefore, it is the larger transverse beam size that helps mitigate the MBI in this arc. Compared with the first example of CCR, the smearing distances $R_{51} \sigma_{x} \approx 10 \mu \mathrm{m}$ and $R_{56} \sigma_{\delta} \approx 30 \mu \mathrm{m}$ are found to be much shorter than the typical microbunched structure at $\lambda \approx 360 \mu \mathrm{m}$, where the maximal gain of CCR occurs. Thus, the absence of effective Landau damping or smearing may be expected and can lead to MBI.

As discussed in Sec. III, the microbunching can be seeded by either initial density or energy modulation. The resultant microbunched structure can reside in the forms of density and energy modulations. Thus the full consideration should be given to the total four types of conversion 

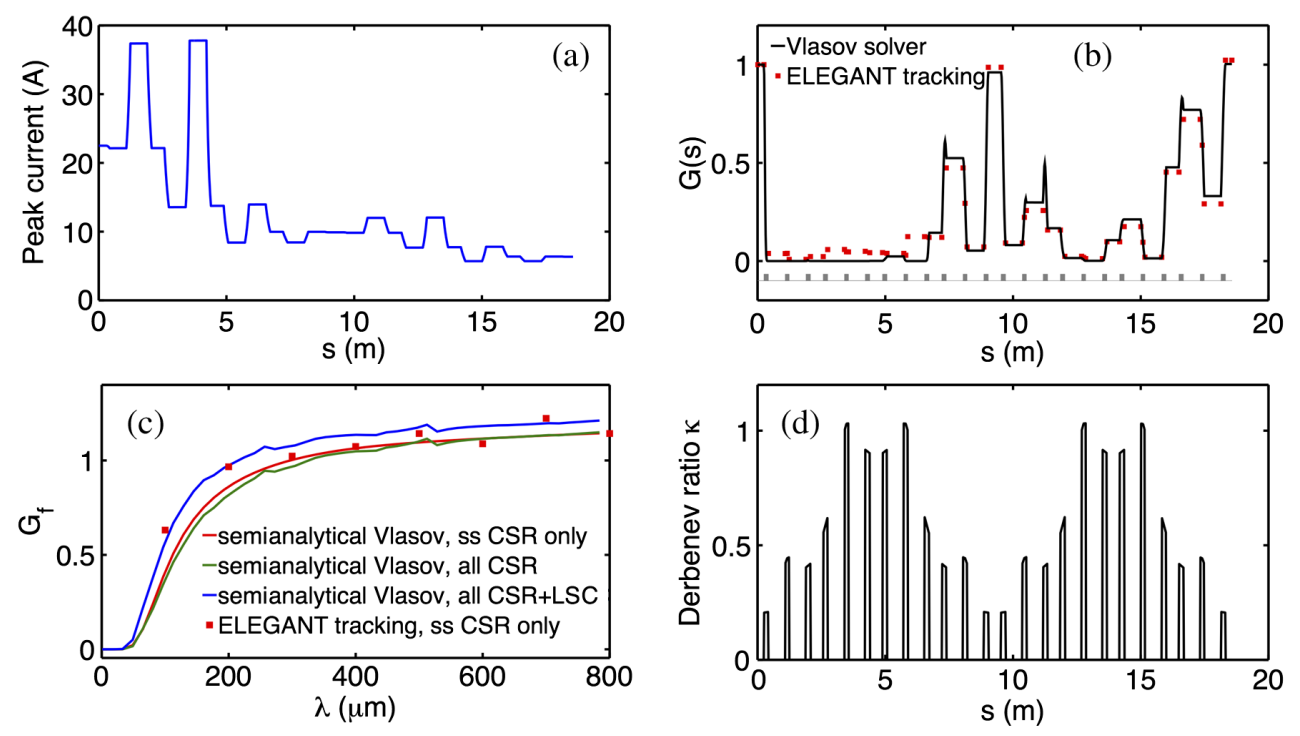

FIG. 6. (a) Bunch decompression along the arc; (b) microbunching gain function $G(s)$ for $\lambda=300 \mu \mathrm{m}$; (c) gain spectrum; (d) Derbenev ratio as a function of $s$. Red dots represent ELEGANT tracking with steady-state (ss) CSR effect.
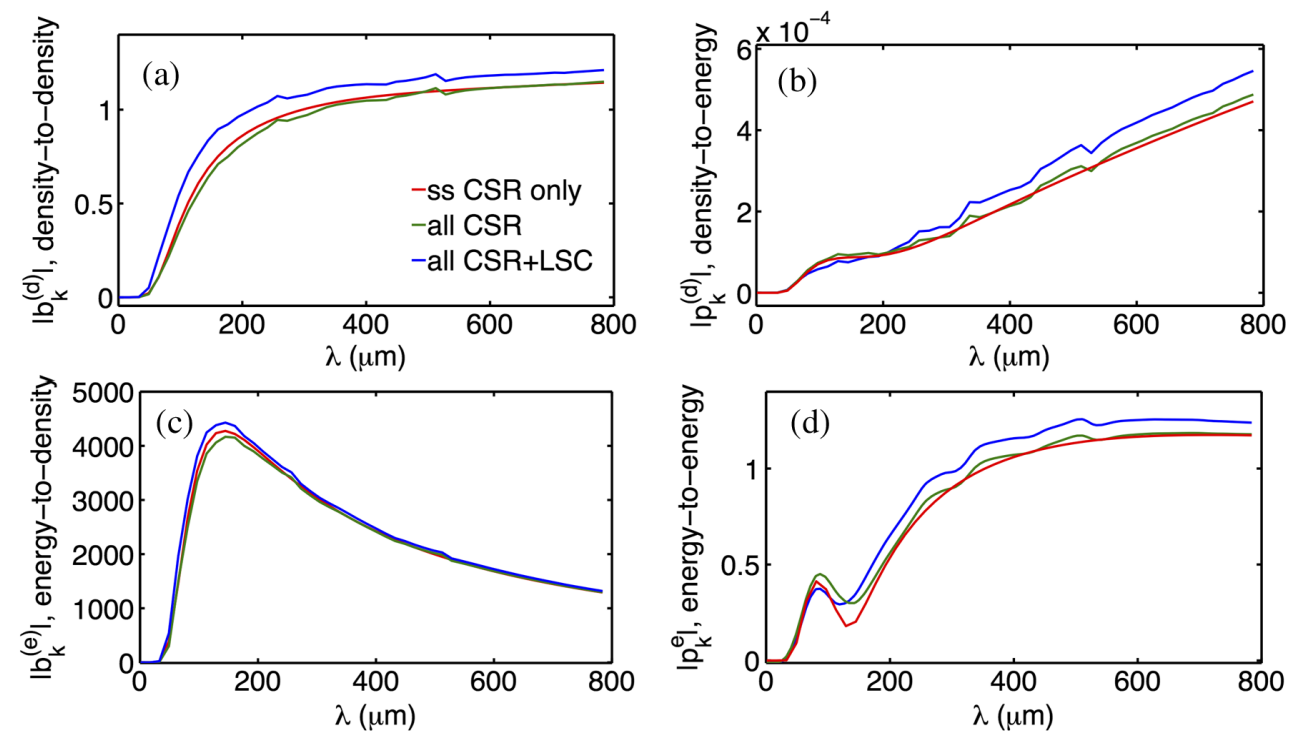

FIG. 7. Microbunching gain spectra for (a) density-to-density; (b) density-to-energy; (c) energy-to-density; and (d) energy-to-energy modulation. Note that in the figures the resultant modulations are evaluated in units of initial modulations.

mechanisms. Shown in Fig. 7, we found the spectral behavior of the modulations is largely from the pure optics of the beam transport. The seemingly large number shown in Fig. 7(c) as energy-to-density ratio may not really cause significant microbunching because the initial energy modulation can be small. Let us presume the energy modulation is of the same order as the uncorrelated energy spread $10^{-4}$ at $150 \mu \mathrm{m}$; the resultant density modulation due to energyto-density conversion at the exit of the arc is 0.4 , which is considered negligible. Our preliminary particle tracking simulation with inclusion of space charge and relevant collective effects shows no evidence of energy modulation in the beam phase space. This better situation, however, requires further start-to-end investigation once a full ring lattice is available.

To end this section, we evaluate the beam current dependence of the maximal microbunching gain for the magnetized beam transport arc of the JLEIC ERL cooler design. For the moment, let us neglect the state-of-art technology that thus far can be achieved in the upstream magnetized gun system and ignore other types of collective effects, and only focus on MBI due to CSR and LSC [Eqs. (57)-(64)]. Figure 8 shows the current dependence of maximum microbunching gain, in which we find the overall gain smaller than two can be retained up to the (peak) bunch current $\sim 100 \mathrm{~A}$, five times higher than 


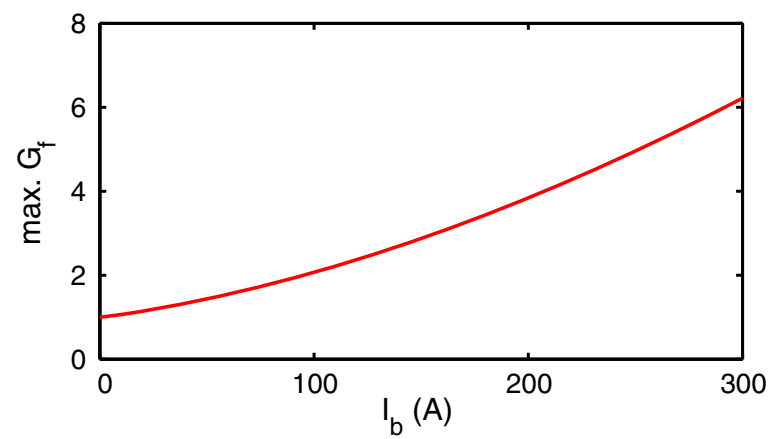

FIG. 8. Initial current dependence of the maximal microbunching gains for the example arc. In the simulation we have included CSR and LSC effects.

the nominal value summarized in Table II. This, to some extent, demonstrates the effectiveness of utilizing the magnetized beam for mitigation of MBI. The advantage of using beam magnetization on microbunching suppression can be seen much more prominently for the strong cooling in next-iterative cooler ring design when the bunch charge is increased.

\section{SUMMARY}

In this paper we have reviewed the single-particle beam optics and applied the concept of sigma matrix to characterize a beam with correlation between any dimensions. Dodging the usage of Twiss (or Courant-Snyder) parametrization in a coupled beam, we take advantage of diagonalizing the beam sigma matrix and find it can greatly simplify the formulation for Vlasov analysis of microbunching for a magnetized beam. A set of governing equations for microbunching analysis of general coupled beams was derived. The theoretical treatment we followed is still largely in the spirit of Refs. [3,4]. Solutions to the integral equations have been found to reduce to the existing well-known formulas for nonmagnetized beams and have been benchmarked against particle tracking simulation. The results all show good agreement. An arc lattice, designed to transport a magnetized beam for downstream ERL electron cooling of the JLEIC, is shown to have nearly no MBI. As a comparison, MBI analysis of the early CCR design is also presented. Suppression of microbunching is found due to effective smearing of relatively large transverse beam size via the transverse-longitudinal correlation $R_{51}$ along the beam line. This smearing takes advantage of a typical feature of beam magnetization. Then a more thorough consideration is taken for both density and energy modulations. More complete analysis will be carried out when a full-ring lattice is available.

Finally we note that our semianalytical Vlasov solver can be used for quick estimates of microbunching in a general coupled beam transport, and for subsequent optimization of beam line design when MBI is a concern. This can be done without tracking a large number of simulation particles [43,54].

\section{ACKNOWLEDGMENTS}

We thank Steve Benson for his coordination of this work and He Zhang for stimulating discussions. One of the authors (C. Y. T.) is thankful for the NAPAC 2016 student grant and his advisor at Virginia Tech, Mark Pitt, for support. This work is supported under U.S. DOE Contract No. DE-AC05-06OR23177.

\section{APPENDIX: GAIN FUNCTION AND SPECTRUM FOR JLEIC CCR WITH $\varepsilon_{n x}=\varepsilon_{n y}=30 \mu \mathrm{m}$}

Because of the ultrahigh gain for the JLEIC CCR with nominal beam parameters (see Table I), to validate our semianalytical solutions against ELEGANT tracking results, we increase the transverse beam emittances to ten times the nominal values, i.e. $\varepsilon_{n x}=\varepsilon_{n y}=30 \mu \mathrm{m}$, so that we can ease the numerical requirements of the number of macroparticles for preparing the initial (modulated) phase-space distributions.

With the increased transverse emittances while keeping other beam and lattice parameters the same, we plot the steady-state CSR gain function $G(s)$ and spectrum $G_{f}(\lambda)$ together with ELEGANT tracking results shown in Figs. 9 and 10. In ELEGANT, $50 \times 10^{6}$ macroparticles were used and 10000 bins employed in simulating CSR effects within dipoles. Both our semianalytical Vlasov results and ELEGANT tracking are in excellent agreement. This validates our semianalytical Vlasov calculation.

Here we have an interesting observation that, in almostno-gain regions, the bunching factors (or, microbunching gains) extracted from particle tracking simulation exhibit some fluctuations. These regions happen to be located at larger dispersive locations; thus transverse coordinates (e.g. $x$ and $x^{\prime}$ ) can be coupled to the longitudinal coordinate $z$ by nonzero energy displacement (via $R_{16}$ and $R_{26}$,

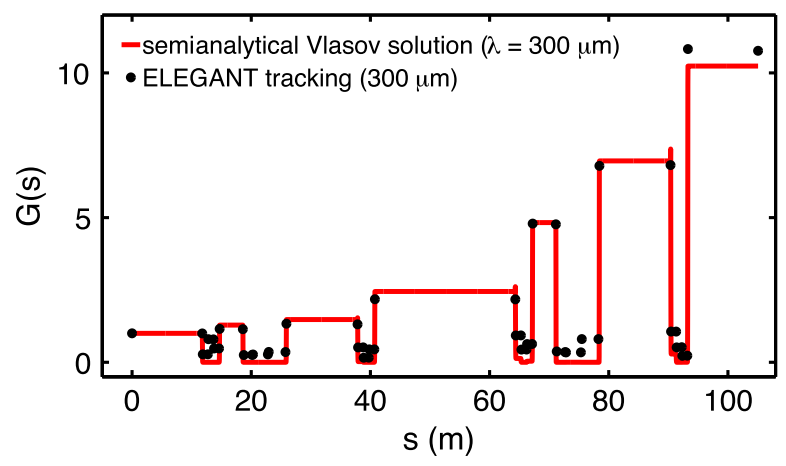

FIG. 9. Steady-state CSR gain functions $G(\mathrm{~s})$ for the JLEIC CCR lattice. Note here that $\lambda=300 \mu \mathrm{m}$ for both the semianalytical solution and ELEGANT tracking. In elegant tracking we impose an initial density modulation amplitude $0.2 \%$ on a flattop density distribution. 


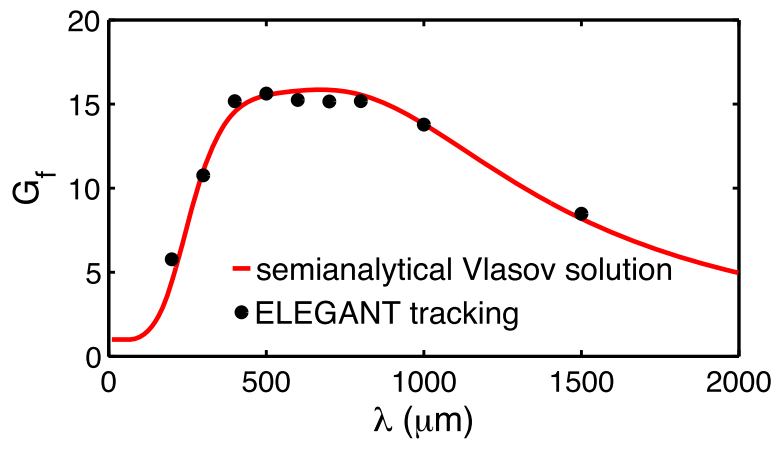

FIG. 10. Steady-state CSR gain spectrum as a function of the initial modulation wavelength for the JLEIC CCR lattice. Here we fix the initial density modulation amplitudes to be $0.2 \%$ for various modulation wavelengths to obtain the final gain spectrum.

or $R_{51}$ and $\left.R_{52}\right)$. In general, $z_{s}=R_{51} x_{0}+R_{52} x_{0}^{\prime}+R_{53} y_{0}+$ $R_{54} y_{0}^{\prime}+z_{0}+R_{56} \delta_{0}$, the nonzero dispersion thus smears out the $z$ coordinate and causes the (projected) bunch distribution to be not as smooth as those at other locations.

[1] M. Borland, in Particle Accelerator Conference 2001 (IEEE, Chicago, IL, USA, 2001), WPPH103.

[2] S. Heifets, G. Stupakov, and S. Krinsky, Coherent synchrotron radiation instability in a bunch compressor, Phys. Rev. ST Accel. Beams 5, 064401 (2002).

[3] Z. Huang and K.-J. Kim, Formulas for coherent synchrotron radiation microbunching in a bunch compressor chicane, Phys. Rev. ST Accel. Beams 5, 074401 (2002).

[4] Ya. Derbenev and Y. Zhang, in Workshop on Beam Cooling and Related Topics (IMP, Lanzhou, China, 2009), FRM2MCCO01.

[5] S. Abeyratne et al., MEIC Design Summary, https://arxiv .org/abs/1504.07961.

[6] C. Tennant and D. Douglas, Report No. JLAB-TN-12-027, 2012.

[7] E. Nissen, Y. Zhang, R. Li, D. Douglas, and C. Tennant, in Proceedings of the International Workshop on Accelerator Science and Technology for Electron-Ion Collider (Jefferson Lab, Newport News, VA, 2014).

[8] C.-Y. Tsai, D. Douglas, R. Li, and C. Tennant, in Proceedings of the International Workshop on Accelerator Science and Technology for Electron-Ion Collider (Jefferson Lab, Newport News, VA, 2014).

[9] C.-Y. Tsai, D. Douglas, R. Li, and C. Tennant, in Proceedings of The 56th ICFA Advanced Beam Dynamics Workshop on Energy Recovery Linacs (Brookhaven National Lab, Stony Brook, New York, 2015), TUICLH2034.

[10] C.-Y. Tsai, D. Douglas, R. Li, and C. Tennant, JLAB Report, No. JLAB-TN-16-030, 2016.

[11] D. R. Douglas, S. V. Benson, A. Hutton, G. A. Krafft, R. Li, G. R. Neil, Y. Roblin, C. D. Tennant, and C.-Y. Tsai, Control of coherent synchrotron radiation and microbunching effects during transport of high brightness electron beams, arXiv:1403.2318v1.
[12] D. R. Douglas, S. V. Benson, A. Hofler, R. Kazimi, R. Li, Y. Roblin, C. D. Tennant, G. A. Krafft, B. Terzic, and C.-Y. Tsai, in Proceedings of the 6th International Particle Accelerator Conference (2015), Richmond, VA (2015), TUPMA038.

[13] C.-Y. Tsai, D. Douglas, R. Li, and C. Tennant, in Proceedings of the 36th International Free Electron Laser Conference (FEL'14), Basel, Switzerland (2014), THP022.

[14] C.-Y. Tsai, D. Douglas, R. Li, and C. Tennant, in Proceedings of the 37th International Free Electron Laser Conference (FEL'15), Daejeon, Korea (2015), MOP087.

[15] C.-Y. Tsai, D. Douglas, R. Li, and C. Tennant, in Proceedings of the 6th International Particle Accelerator Conference (2015), Richmond, VA (2015), MOPMA028.

[16] C.-Y. Tsai, S. Di Mitri, D. Douglas, R. Li, and C. Tennant, Proceedings of the 7th International Particle Accelerator Conference, Busan, Korea (2016), TUOAB02.

[17] Ya. S. Derbenev and I. Meshkov, CERN Report No. 77-08, 1977.

[18] Ya. S. Derbenev and A. N. Skrinsky, The effect of an accompanying magnetic field on electron cooling, Part. Accel. 8, 235 (1978).

[19] Ya. Derbenev, MEIC Spring Collaboration Meeting, 2015 (unpublished).

[20] K.-J. Kim, Round-to-flat transformation of angularmomentum-dominated beams, Phys. Rev. ST Accel. Beams 6, 104002 (2003).

[21] H. Zhang, D. Douglas, Ya. Derbenev, and Y. Zhang, Proceedings of the 6th International Particle Accelerator Conference, Richmond, VA (2015), TUPWI037.

[22] A. V. Fedotov, I. Ben-Zvi, D. L. Bruhwiler, V. N. Litvinenko, and A. O. Sidorin, High-energy electron cooling in a collider, New J. Phys. 8, 283 (2006).

[23] V. Parkhomchuk and I. Ben-Zvi, Report No. C-A/AP/47, 2001.

[24] Ya. Derbenev, A method to overcome space charge at injection, AIP Conf. Proc. 773, 335 (2005).

[25] V. A. Lebedev and S. A. Bogacz, Betatron motion with coupling of horizontal and vertical degrees of freedom, J. Instrum. 5, P10010 (2010).

[26] M. Borland, APS Light Source Note LS-287, 2000.

[27] G. Stupakov, Lecture notes on Classical Mechanics and Electromagnetism in Accelerator Physics, The U.S. Particle Accelerator School (2011).

[28] A. J. Dragt, R. L. Gluckstern, F. Neri, and G. Rangarajan, in Frontiers of particle beams; observation, diagnosis and correction, Lect. Notes Phys. 343, 94 (1988).

[29] R. Q. Twiss and N. H. Frank, Orbital stability in a proton synchrotron, Rev. Sci. Instrum. 20, 1 (1949).

[30] E. D. Courant and H. S. Snyder, Theory of the alternatinggradient synchrotron, Ann. Phys. (N.Y.) 3, 1 (1958).

[31] A. Chao, SLAC Report No. SLAC-PUB-9574, 2002, http://www.slac.stanford.edu/ achao/lecturenotes.html.

[32] A. A. Vlasov, On vibration properties of electron gas, J. Exp. Theor. Phys. 8, 291 (1938).

[33] Note that the coasting beam approximation is no longer valid when a beam is critically compressed or experiences parasitic compression. The definitions of the "parasitic compression" and the "critical compression" were from D. Douglas (see also Ref. [53]). 
[34] R. Li and C.-Y. Tsai, in Proceedings of the 6th International Particle Accelerator Conference, Richmond, VA (2015), MOPMN004.

[35] J. B. Murphy, S. Krinsky, and R. L. Gluckstern, Longitudinal wakefield for an electron moving on a circular orbit, Part. Accel. 57, 9 (1997).

[36] Ya. S. Derbenev, J. Rossbach, E. L. Saldin, and V. D. Shiltsev, TESLA-FEL-Report No. 1995-05.

[37] D. Zhou, in Proceedings of the 3rd International Particle Accelerator Conference, New Orleans, Louisiana, USA, 2012 (IEEE, Piscataway, NJ, 2012), MOOBB03.

[38] C. Mitchell and J. Qiang, in Proceedings of the 4th International Particle Accelerator Conference, Shanghai, China (2013), TUPWA057.

[39] E. L. Saldin, E. A. Schneidmiller, and M. V. Yurkov, On the coherent radiation of an electron bunch moving in an arc of a circle, Nucl. Instrum. Methods Phys. Res., Sect. A 398, 373 (1997).

[40] R. Li and C.-Y. Tsai, Entrance and Exit CSR Impedance for Non-ultrarelativistic Beams (unpublished).

[41] M. Venturini, Models for longitudinal space charge impedance for microbunching instability, Phys. Rev. ST Accel. Beams 11, 034401 (2008).

[42] J. Wu, Z. Huang, and P. Emma, Analytical Analysis of longitudinal space charge effects for a bunched beam with radial dependence, Phys. Rev. ST Accel. Beams 11, 040701 (2008).
[43] C.-Y. Tsai, D. Douglas, R. Li, and C. Tennant, Linear microbunching analysis for recirculation machines, Phys. Rev. Accel. Beams 19, 114401 (2016).

[44] S. Abeyratne et al., Science Requirements and Conceptual Design for a Polarized Medium Energy Electron-Ion Collider at Jefferson Lab, https://arxiv.org/abs/1209.0757 (2012).

[45] S. Benson, Requirements and Status of Bunched Beam ERL Cooler Design, JLEIC Collaboration Meeting (2016).

[46] M. Borland, Report No. OAG-TN-2005-027.

[47] A. Burov, S. Nagaitsev, A. Shemyakin, and Ya. Derbenev, Optical principles of beam transport for relativistic electron cooling, Phys. Rev. ST Accel. Beams 3, 094002 (2000).

[48] D. Douglas and C. Tennant, JLAB Report No. JLAB-TN15-026.

[49] D. Douglas, JLAB Report No. JLAB-TN-15-022.

[50] M. Borland, Modeling the microbunching instability, Phys. Rev. ST Accel. Beams 11, 030701 (2008).

[51] C.-Y. Tsai and R. Li, JLAB Report No. JLAB-TN-14-016.

[52] Ya. Derbenev and V. Shiltsev, SLAC Report No. SLACPUB-7181 1996.

[53] R. Li, Curvature-induced bunch self-interaction for an energy-chirped bunch in magnetic bends, Phys. Rev. ST Accel. Beams 11, 024401 (2008).

[54] For example, each dot in Fig. 6(c) (from ELEGANT tracking) can take about $3 \mathrm{~h}$ using a single CPU in a personal desktop computer. For our semianalytical Vlasov solver (written in MATLAB), it only takes seconds on the same computer. 